\title{
Quandle homotopy invariants of knotted surfaces
}

\author{
Takefumi Nosaka
}

Received: 17 February 2012 / Accepted: 22 June 2012 / Published online: 10 October 2012

(C) The Author(s) 2012. This article is published with open access at Springerlink.com

\begin{abstract}
Given a finite quandle, we introduce a quandle homotopy invariant of knotted surfaces in the 4-sphere, modifying that of classical links. This invariant is valued in the third homotopy group of the quandle space, and is universal among the (generalized) quandle cocycle invariants. We compute the second and third homotopy groups, with respect to "regular Alexander quandles". As a corollary, any quandle cocycle invariant using the dihedral quandle of prime order is a scalar multiple of Mochizuki 3-cocycle invariant. As another result, we determine the third quandle homology group of the dihedral quandle of odd order.
\end{abstract}

Keywords Quandle $\cdot$ Classical link $\cdot$ Knotted surface $\cdot$ Rack space . Quandle cocycle invariant $\cdot k$-invariant $\cdot$ Homotopy group $\cdot$ Loop space

\section{Introduction}

A quandle is an algebraic system satisfying axioms that correspond to the Reidemeister moves. Given a quandle $X$, Fenn et al. [13] defined the rack space $B X$, in analogy to the classifying spaces of groups; Further, an invariant of framed links in $S^{3}$ was proposed in $[14, \S 4]$ which is called a quandle homotopy invariant and is valued in the second homotopy group $\pi_{2}(B X)$ [see [23] for some computations of $\pi_{2}(B X)$ ]. In addition, as a modification of the homology $H_{*}(B X ; \mathbb{Z})$, Carter et al. [4] introduced its quandle homology denoted by $H_{n}^{Q}(X ; A)$, and further quandle cocycle invariants of classical links (resp. linked surfaces) using cohomology classes in $H_{Q}^{2}(X ; A)$ (resp. $\left.H_{Q}^{3}(X ; A)\right)$. For its applications, the homology groups $H_{*}(B X ; A)$ and $H_{*}^{Q}(X ; A)$ of some quandles $X$ have been computed $[5,8,19,20,24,26]$. Furthermore the quandle cocycle invariants were generalized to allow the cohomology $H_{Q}^{*}(X ; A)$ with local coefficients [3].

T. Nosaka ( $\square)$

Research Institute for Mathematical Sciences, Kyoto University,

Sakyo-ku, Kyoto 606-8502, Japan

e-mail: nosaka@kurims.kyoto-u.ac.jp 
As for classical links, the quandle cocycle invariants were much studied (see, e.g., $[15,23,25,31])$, and are known to be derived from the quandle homotopy invariant above (see $[6,31]$ ). The study of the homotopy group $\pi_{2}(B X)$ was useful to understood a topological meaning of some quandle cocycle invariants [15].

In this paper, we introduce and study a quandle homotopy invariant of oriented linked surfaces valued in a group ring $\mathbb{Z}\left[\pi_{3}^{Q}(B X)\right]$ (Definition 2.3), modifying the above quandle homotopy invariant of links in $S^{3}$. Here the group $\pi_{3}^{Q}(B X)$ is defined by a certain quotient group of the third homotopy group $\pi_{3}(B X)$ (see Remark 2.2 for details). Similar to the quandle homotopy invariant of classical links, that of linked surfaces is shown to be universal among the quandle cocycle invariants with local coefficients of linked surfaces (see Sect. 2.3). It is therefore significant to estimate and determine $\pi_{3}^{Q}(B X)$. Moreover, from the study of $\pi_{3}^{Q}(B X)$, we address a problem of determining those local coefficients for which the the associated quandle cocycle invariants pick out completely the quandle homotopy invariant.

First, we determine the free subgroup of $\pi_{3}^{Q}(B X)$ of a finite quandle $X$, using rational homotopy theory (Theorem 3.1). We show that $\pi_{3}^{Q}(B X)$ is finitely generated, and that $\pi_{3}^{Q}(B X) \otimes \mathbb{Q}$ depends only on the number $\ell$ of "the connected components" of $X$ : To be precise, $\pi_{3}^{Q}(B X) \otimes \mathbb{Q} \cong \mathbb{Q}^{\ell(\ell-1)(\ell-2) / 3}$. Further, we give a topological interpretation of $\pi_{3}^{Q}(B X) \otimes \mathbb{Q}$ from a view of "link bordism groups" (see Remark 4.2).

Next we deal with the torsion subgroups of $\pi_{3}^{Q}(B X)$. Since it is difficult to compute $\pi_{3}^{Q}(B X)$ in general, in this paper, we confine ourselves to regular Alexander quandles $X$. Here the regular Alexander quandle $X$ is defined to be a $\mathbb{Z}\left[T^{ \pm 1}\right]$-module with a binary operation $(x, y) \mapsto T x+(1-T) y$ which satisfies $(1-T) X=X$ and the minimal $e$ satisfying $\left(T^{e}-1\right) X=0$ is relatively prime to $|X|$.

In this case, we estimate $\pi_{3}^{Q}(B X)$ roughly: When $X$ is a regular Alexander quandle of odd order, we obtain an exact sequence which estimates $\pi_{3}^{Q}(B X)$ from upper bounds by the homologies $H_{j}(B X ; \mathbb{Z})$ with $j \leq 4$ (Proposition 3.4). However, under an additional assumption, we succeed in determining $\pi_{3}^{Q}(B X)$ in a homological context. Specifically, if $H_{2}^{Q}(X ; \mathbb{Z})$ vanishes and $|X|$ is odd, then $\pi_{3}^{Q}(B X)$ is isomorphic to $H_{4}^{Q}(X ; \mathbb{Z})$ (Theorem 3.5). We here remark that there are many such quandles, e.g., the Alexander quandle of the form $X=\mathbb{F}_{q}[T] /(T-\omega)$ where $\mathbb{F}_{q}$ is the finite field of order $p^{2 h-1}$ and $\omega \in \mathbb{F}_{q} \backslash\{0, \pm 1\}$ (see Remark 3.6). In particular, we determine $\pi_{3}^{Q}(B X)$ of all Alexander quandles $X$ of prime order (see Corollary 3.7), using the homology $H_{4}^{Q}(X ; \mathbb{Z})$ determined in [23]. As the simplest case, if $X=\mathbb{Z}[T] /(p, T+1)$ which is called the dihedral quandle, then $\pi_{3}^{Q}(B X) \cong \mathbb{Z} / p \mathbb{Z}$ and any quandle cocycle invariant of $X$ turns out to be a scalar multiple of the quandle cocycle invariant $\Psi_{\theta_{p}}(L) \in \mathbb{Z}\left[\mathbb{F}_{p}\right]$ of a 3-cocycle $\theta_{p} \in H_{Q}^{3}\left(X ; \mathbb{F}_{p}\right)$ that is called Mochizuki 3-cocycle (Corollary 3.8). In summary, the study to compute $\pi_{3}^{Q}(B X)$ picks out all non-trivial quandle cocycle invariants defined via $X$.

In another direction, by a similar discussion, we also compute the second homotopy group $\pi_{2}(B X)^{1}$ with respect to regular Alexander quandles $X$. Recall that $\pi_{2}(B X)$ is the container of the quandle homotopy invariant of framed links. Our result on $\pi_{2}(B X)$ is that, if $X$ is of odd order, the following exact sequence splits (Theorem 3.9):

$$
0 \longrightarrow \pi_{2}(B X) \longrightarrow \mathbb{Z} \oplus H_{3}^{Q}(X ; \mathbb{Z}) \longrightarrow H_{2}^{Q}(X ; \mathbb{Z}) \wedge_{\mathbb{Z}} H_{2}^{Q}(X ; \mathbb{Z}) \longrightarrow 0 .
$$

$1 \pi_{2}(B X) \cong \mathbb{Z} \oplus \pi_{2}\left(B X_{Q}\right)$ is known [23], where $B X_{Q}$ is the quandle space. In $\S 3$ and 4 , we mainly deal with $\pi_{2}\left(B X_{Q}\right)$. 
Table 1 Some homotopy groups $\pi_{2}\left(B X_{Q}\right)$ obtained from $H_{2}^{Q}(X ; \mathbb{Z})$ and $H_{3}^{Q}(X ; \mathbb{Z})$

\begin{tabular}{llll}
\hline$X$ & $H_{2}^{Q}(X ; \mathbb{Z})$ & $H_{3}^{Q}(X ; \mathbb{Z})$ & $\pi_{2}\left(B X_{Q}\right)$ \\
\hline $\mathbb{Z}[T] /(5, T+1)$ & 0 & $\mathbb{Z} / 5 \mathbb{Z}$ & $\mathbb{Z} / 5 \mathbb{Z}$ \\
$\mathbb{Z}[T] /(5, T-\omega)$ & 0 & 0 & 0 \\
$\mathbb{Z}[T] /(7, T+1)$ & 0 & $\mathbb{Z} / 7 \mathbb{Z}$ & $\mathbb{Z} / 7 \mathbb{Z}$ \\
$\mathbb{Z}[T] /(7, T-\omega)$ & 0 & 0 & 0 \\
$\mathbb{Z}[T] /\left(2, T^{3}+T^{2}+1\right)$ & 0 & $\mathbb{Z} / 2 \mathbb{Z}$ & $\mathbb{Z} / 2 \mathbb{Z}$ \\
$\mathbb{Z}[T] /\left(2, T^{3}+T+1\right)$ & 0 & $\mathbb{Z} / 2 \mathbb{Z}$ & $\mathbb{Z} / 2 \mathbb{Z}$ \\
$\mathbb{Z}[T] /(9, T+1)$ & 0 & $\mathbb{Z} / 9 \mathbb{Z}$ & $\mathbb{Z} / 9 \mathbb{Z}$ \\
$\mathbb{Z}[T] /\left(3, T^{2}+1\right)$ & $\mathbb{Z} / 3 \mathbb{Z}$ & $(\mathbb{Z} / 3 \mathbb{Z})^{3}$ & $(\mathbb{Z} / 3 \mathbb{Z})^{3}$ \\
$\mathbb{Z}[T] /\left(3, T^{2}+T-1\right)$ & 0 & 0 & 0 \\
$\mathbb{Z}[T] /\left(3, T^{2}-T-1\right)$ & 0 & 0 & 0 \\
\hline
\end{tabular}

Here $\omega \neq \pm 1$

In particular, $\pi_{2}(B X)$ is a direct summand of $\mathbb{Z} \oplus H_{3}^{Q}(X ; \mathbb{Z})$. In conclusion, regarding $H_{3}^{Q}(X ; \mathbb{Z})$ as a second homology with local coefficients (see Remark 4.1), Theorem 3.9 implies that the quandle homotopy invariant is completely determined as a linear sum of quandle cocycle invariants through $H_{Q}^{2}(X ; A) \oplus H_{Q}^{3}(X ; A)$ of the local system (Remark 5.5).

Furthermore, the above sequence enables us to compute $\pi_{2}(B X)$. For several quandles $X$ of order $\leq 9$, we determine $\pi_{2}(B X)$ exactly (see Table 1 ), following the values of $H_{2}^{Q}(X ; \mathbb{Z})$ and $H_{3}^{Q}(X ; \mathbb{Z})$ presented in [17]. Furthermore, since Mochizuki [19,20] has computed $H_{Q}^{2}\left(X ; \mathbb{F}_{q}\right) \oplus H_{Q}^{3}\left(X ; \mathbb{F}_{q}\right)$ for $X=\mathbb{F}_{q}[T] /(T-\omega)$, we determine $\pi_{2}(B X) \otimes_{\mathbb{Z}} \mathbb{F}_{p}$ and have made the quandle homotopy invariants computable (see Appendix A). One of the noteworthy results is that if $X$ is the product $h$-copies of the dihedral quandle, i.e., $X=(\mathbb{Z}[T] /(p, T+1))^{h}$, then the dimension of $\pi_{2}(B X) \otimes_{\mathbb{Z}} \mathbb{F}_{p}$ is a quadratic function of $h$ : to be precise, $\operatorname{dim}_{\mathbb{F}_{p}}\left(\pi_{2}(B X) \otimes_{\mathbb{Z}} \mathbb{F}_{p}\right)=1+\left(h^{2}\left(h^{2}+11\right) / 12\right)$ [Corollary A.4].

As a corollary of our work, for an odd $m$, we determine $H_{3}^{Q}(X ; \mathbb{Z})$ of the dihedral quandle $X$ of order $m$, i.e., $X=\mathbb{Z}[T] /(m, T+1)$. Since Hatakenaka and the author [15] computed $\pi_{2}(B X) \cong \mathbb{Z} \oplus \mathbb{Z} / m$, it follows from Theorem 3.9 above that $H_{3}^{Q}(X ; \mathbb{Z}) \cong \mathbb{Z} / m$ (Corollary 3.11). When $m$ is an odd prime, the isomorphism $H_{3}^{Q}(X ; \mathbb{Z}) \cong \mathbb{Z} / m$ was conjectured by Fenn, Rourke and Sanderson (see [27, Conjceture 5.12]) and solved by Niebrzydowski and Przytycki using homological algebra [25]. On the other hand, our proof is another approach from $\pi_{2}(B X)$, and further gives a generalization of the conjecture.

This paper is organized as follows. In Sect. 2, we review quandles and the rack spaces $B X$, and define the quandle homotopy invariant. In Sect. 3, we state our theorems and corollaries. In Sect. 4, we review the quandle homology groups, and prove Theorem 3.1 on the rational homotopy group $\pi_{3}^{Q}(B X) \otimes \mathbb{Q}$. In Sect. 5, we show Theorems 3.5 and 3.9. In Appendix A, we compute $\pi_{2}(B X) \otimes \mathbb{Z} / p \mathbb{Z}$ for some Alexander quandles $X$ on finite fields.

Notational convention Throughout this paper, $\mathbb{F}_{q}$ is a finite field of characteristic $p>0$. We denote by $\mathbb{Z}_{m}$ the cyclic group of order $m \in \mathbb{Z}$. A symbol ' $\otimes$ ' is always the tensor product over $\mathbb{Z}$. For a group $G$, we denote by $\mathbb{Z}[G]$ the group ring of $G$ over $\mathbb{Z}$. For a finitely generated $\mathbb{Z}$-module $N, N_{(p)}$ means the localization at a prime $p$. Further, $\Lambda^{*}(N)$ denotes the $*$-part of the exterior algebra over $N$. A symbol 'pt.' stands for a single point.

All embeddings of surfaces into $S^{4}$ are assumed to be oriented, closed and of $C^{\infty}$-class. 


\section{Quandle homotopy invariants of linked surfaces}

We review quandles and the rack spaces in Sect. 2.1, and introduce quandle homotopy invariants of linked surfaces in Sect. 2.2.

\subsection{Review of quandles and of rack spaces}

A quandle is a set $X$ with a binary operation $(x, y) \rightarrow x * y$ such that, for any $x, y, z \in X$, $x * x=x,(x * y) * z=(x * z) *(y * z)$ and there exists a unique element $w \in X$ such that $w * y=x$. For example, a $\mathbb{Z}\left[T^{ \pm 1}\right]$-module $M$ has a quandle structure given by $x * y:=T x+(1-T) y$, called an Alexander quandle. For a quandle $X$, the associated group of $X$ is defined by the group presentation $\operatorname{As}(X):=\langle x \in X \mid x \cdot y=y \cdot(x * y)(x, y \in X)\rangle$. For any quandle $X, \operatorname{As}(X)$ is of infinite order, since $\operatorname{As}(X)$ has an epimorphism onto $\mathbb{Z}$ obtained by the length of words. An $X$-set is defined to be a set $Y$ acted on by $\operatorname{As}(X)$. For example, when $Y=X$, the quandle $X$ is itself an $X$-set by means of the quandle operation.

We next review $X$-colorings [4, Definition 5.1.]. Let $D$ be a broken diagram of a linked surface $L$. In this paper, any broken diagram is regarded being in $S^{3}$. An $X$-coloring is a map $C$ from the set of sheets of $D$ to $X$ such that, for every double-point curve as shown in the left of Fig. 1, the three sheets $\alpha, \beta, \gamma$ satisfy $C(\gamma)=C(\alpha) * C(\beta)$. This definition is compatible with triple-points (see the right of Fig. 1). We denote the set consisting of such $X$-colorings of $D$ by $\operatorname{Col}_{X}(D)$. It is widely known (see, e.g., [4, Theorem 5.6]) that if two diagrams $D$ and $D^{\prime}$ are related by the Roseman moves, then there naturally exists a 1-1 correspondence between $\operatorname{Col}_{X}(D)$ and $\mathrm{Col}_{X}\left(D^{\prime}\right)$. Furthermore, when $X$ is an Alexander quandle of the form $X=\mathbb{Z}_{p}\left[T^{ \pm 1}\right] /(h(T))$ and $L$ is a knotted surface, the set $\operatorname{Col}_{X}(D)$ is obtained from the Alexander polynomials of $L$ (see [16] for details).

Finally, we review the rack space introduced by Fenn-Rourke-Sanderson [13]. Fix an $X$-set $Y$. Equipping $X$ and $Y$ with their discrete topology, we start with $\bigcup_{n \geq 0}(Y \times([0,1] \times$ $X)^{n}$ ), and consider the equivalence relations given by

$$
\begin{aligned}
& \left(y ; t_{1}, x_{1}, \ldots, x_{j-1}, 1, x_{j}, t_{j+1}, \ldots, t_{n}, x_{n}\right) \\
& \quad \sim\left(y \cdot x_{j} ; t_{1}, x_{1} * x_{j}, \ldots, t_{j-1}, x_{j-1} * x_{j}, t_{j+1}, x_{j+1}, \ldots, t_{n}, x_{n}\right), \\
& \left(y ; t_{1}, x_{1}, \ldots, x_{j-1}, 0, x_{j}, t_{j+1}, \ldots, t_{n}, x_{n}\right) \\
& \quad \sim\left(y ; t_{1}, x_{1}, \ldots, t_{j-1}, x_{j-1}, t_{j-1}, x_{j+1}, \ldots, t_{n}, x_{n}\right) .
\end{aligned}
$$

Here our description divides the element of $Y$ from the elements of $([0,1] \times X)^{n}$ by a semicolon ';'. Then the rack space, $B X^{Y}$, is defined by the quotient space (we later explain the 3-skeleton for more details in Sect. 2.2). By construction, we have a cell decomposition of
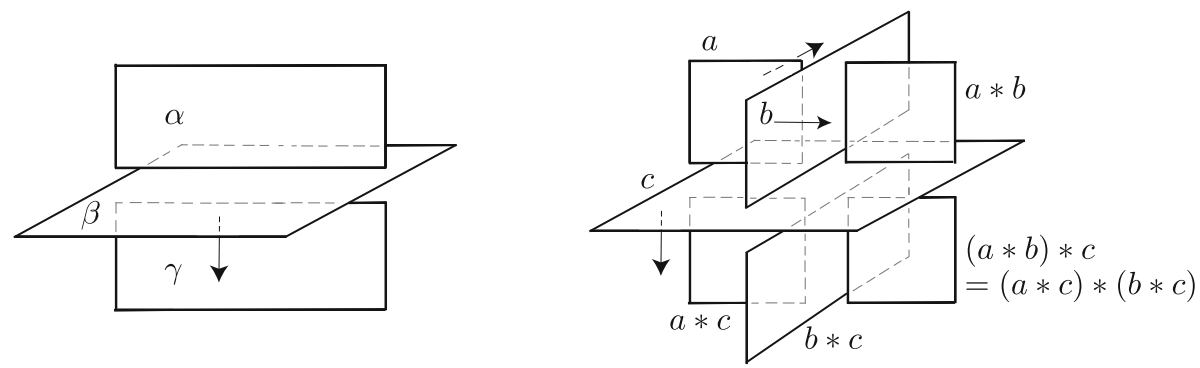

Fig. 1 Coloring conditions around double-point curves and triple-points. Here $a, b, c \in X$ 
$B X^{Y}$, by regarding the projection $\bigcup_{n \geq 0}\left(Y \times([0,1] \times X)^{n}\right) \rightarrow B X^{Y}$ as the characteristic maps. We easily see that if the action on $Y$ of $\operatorname{As}(X)$ is transitive, the space $B X^{Y}$ is pathconnected (see [8, Proposition 2.12] for details). When $Y$ is a single point, we denote the path-connected space $B X^{Y}$ by $B X$ for short. Note that $\pi_{1}(B X) \cong$ As $(X)$ by the definition of the 2-skeleton of $B X$.

\subsection{Definition of a quandle homotopy invariant of linked surfaces}

Our purpose is to define a quandle homotopy invariant of linked surfaces. This is an analogue of the quandle homotopy invariant of (framed) links in $S^{3}[14, \S 3]$ (see also [23, §2]). For the purpose, given an $X$-set $Y$, we first define a quandle space as follows. Define a subspace by

$$
\bigcup_{n \geq 2}\left\{\left(y ; t_{1}, x_{1}, \ldots, t_{n}, x_{n}\right) \in Y \times([0,1] \times X)^{n} \mid x_{i}=x_{i+1} \text { for some } i \in \mathbb{Z}, i \leq n-1\right\} \text {. }
$$

We denote this by $X_{D}^{Y}$, and consider a composite $\iota_{D}^{Y}: X_{D}^{Y} \hookrightarrow \bigcup_{n \geq 0}\left(Y \times([0,1] \times X)^{n}\right) \rightarrow$ $B X^{Y}$. Here the second map is the projection mentioned above.

Definition 2.1 Let $X$ be a quandle. Let $Y$ be an $X$-set. We define a space $B X_{Q}^{Y}$ as the cone of the composite map $\iota_{D}^{Y}: X_{D}^{Y} \rightarrow B X^{Y}$. We refer to the space as quandle space.

When $Y$ is a single point, we denote $B X_{Q}^{Y}$ by $B X_{Q}$ for short. The homology $H_{*}\left(B X_{Q} ; \mathbb{Z}\right)$ coincides with the homology of the quandle complex introduced in [4] (see also Sect. 4.1). Roughly speaking, the space $B X_{Q}$ is a geometric realization of the quandle complex.

We now describe the 3-skeleton of the rack space $B X$ and the 4-skeleton of the quandle space $B X_{Q}$ in more details. The 1-skeleton of $B X$ is a bouquet of $|X|$-circles labeled by elements of $X$. The 2-skeleton of $B X$ is obtained from the 1-skeleton by attaching 2-cells of squares labeled by $(a, b)$ for any $a, b \in X$, where the 4 edges with $X$-labels as shown in Fig. 2 are attached to the corresponding 1-cells. In addition, we attach $\left|X^{3}\right|$-cubes labeled by $(a, b, c) \in X^{3}$, whose six faces are labeled as shown in Fig. 2, to the corresponding 2-cells. The resulting space is the 3 -skeleton of $B X$.

We next describe the 4-skeleton of $B X_{Q}$. To begin, the 3-skeleton of $B X_{Q}$ is obtained from the 3-skeleton of $B X$ by attaching 3-cells which bound 2-cells labeled by $(a, a)$ for $a \in X$. The 4-skeleton of $B X_{Q}$ is obtained from the 3-skeleton of $B X_{Q}$ by attaching 4-cells as follows: for $a, b \in X$, a 3 -cell labeled by either $(a, a, b)$ or $(a, b, b)$ is bounded by a 4cell, and the 4-dimensional cube labeled by $(a, b, c, d) \in X^{4}$ bounds the eight corresponding
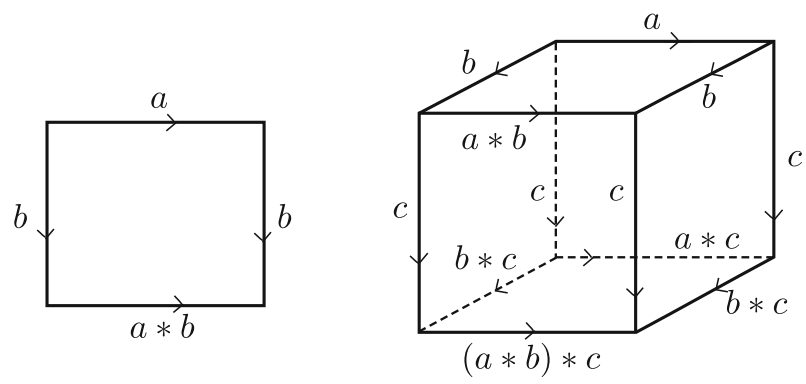

Fig. 2 The 2-cell labeled by $(a, b)$ and the 3-cell labeled by $(a, b, c)$ 


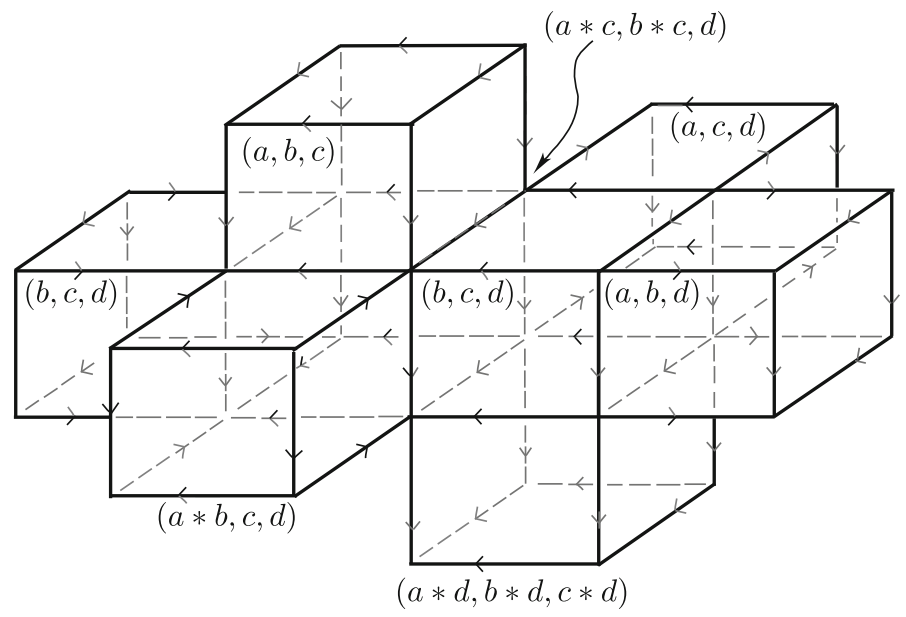

Fig. 3 The eight cubes with labels as the boundary of the 4-cell labeled by $(a, b, c, d)$
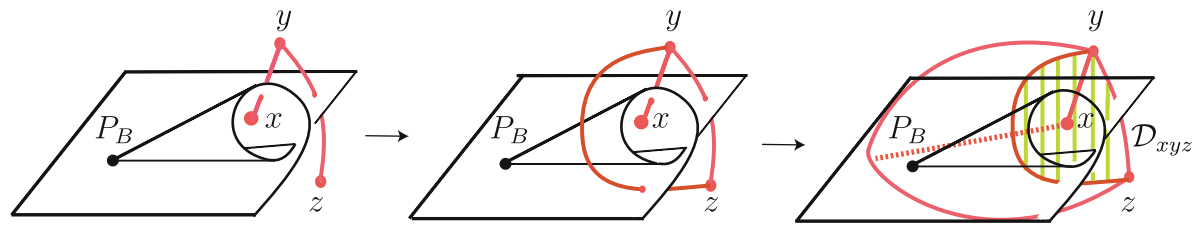

Fig. 4 The modification of the dual decomposition I

3 -cells as shown in Fig. 3. Note that the preceding quandle space considered in $[23, \S 2]^{2}$ is the 3-skeleton of the space $B X_{Q}$, and the higher skeleton was not defined in [23].

In order to construct an invariant of linked surfaces, we prepare a cell decomposition of $S^{3}$. Let $L \subset S^{4}$ be a linked surface. We fix a broken surface diagram $D \subset S^{3}$ of $L$. Regarding $D$ as a decomposition of $S^{3}$ by an immersed surface, we consider the dual decomposition. Recall that the broken diagram is locally composed of some double-point curves, branch-points and triple-points (see, e.g., [9] [4, §5] for the definitions). We then modify the dual decomposition around each branch-points $P_{B}$ of $D$ as follows. To begin, we add an interval which connects between $y$ and $z$ shown on the left of Fig. 4. Put a square $\mathcal{D}_{x y z}$ which is bounded by the three intervals, and further attach a 3-cell which is bounded by the square $\mathcal{D}_{x y z}$ and contains the branch-point $P_{B}$ (see the right of Fig. 4); we here notice that the 3-cell forms a cone on a square. In addition, we attach a cube to the square $\mathcal{D}_{x y z}$ from the opposite direction. For example, if the Whitney umbrella crosses another sheet such as the left of Fig. 5, we here attach 2-cells and 3-cells to the dual decomposition shown as the right of Fig. 5. We orient the modified cell decomposition by using the orientations of $D$.

Next, given an $X$-coloring $C$ of $D$, we will construct a map $\xi_{D, C}: S^{3} \rightarrow B X_{Q}$, as follows. We take the 0 -cells of the modified decomposition to the single 0 -cell of $B X$. We send the 1-cells at sheets colored by $a$ to the 1-cell labeled by $a$. We take the 2-cells around the double-point curves colored by $(a, b)$ to the 2-cell labeled by $(a, b)$. We map the 3-cells around the triple-points colored by $(a, b, c)$ to the 3-cell labeled by $(a, b, c)$ as shown in Fig. 6. Furthermore, we take the modified 3-cells around the branch-points colored by $a \in X$

$\widehat{2}$ In [23], the rack (resp. quandle) space was denoted by $\widehat{B X}$ (resp. $B X$ ). 

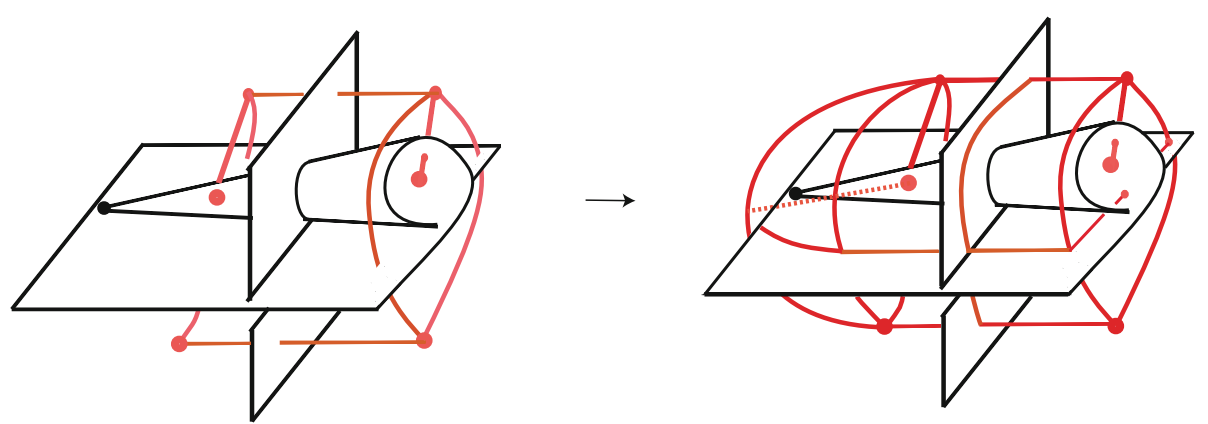

Fig. 5 The modification of the dual decomposition II
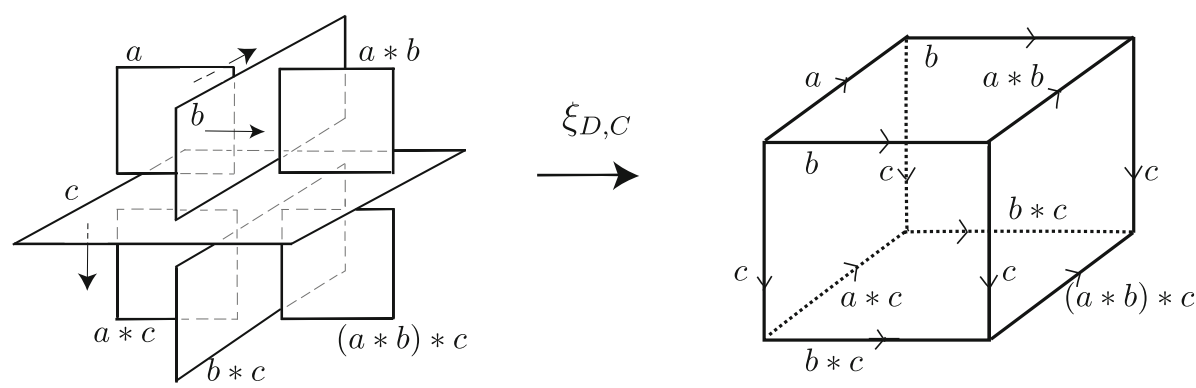

Fig. 6 From a triple-point colored by $(a, b, c)$ to the 3-cell labeled by $(a, b, c)$

to the above cone on the 2-cell labeled by $(a, a)$ (see the right of Fig. 4). By collecting them, we obtain a cellular map $\xi_{D, C}: S^{3} \rightarrow B X_{Q}$. We denote the homotopy class of $\xi_{D, C}$ by $\Xi_{X}(D ; C) \in \pi_{3}\left(B X_{Q}\right)$. By the construction of 4-cells of $B X_{Q}$, we can verify that the homotopy class $\Xi_{X}(D ; C)$ is invariant under the Roseman moves (cf. [4, Theorem 5.6]). For example, the invariance of the move in the bottom right of [4, Figure 7] corresponds to the 4-cell in Fig. 3.

Remark 2.2 It is known that any oriented surface-link is represented by a broken diagram $D$ without branch-points (see [9]). Recall that we defined the group $\pi_{3}^{Q}(B X)$ to be the quotient of $\pi_{3}(B X)$ by a normal subgroup that takes account of branch-points of knotted surfaces. However, for such a diagram $D$, we claim that the map $\xi_{D, C}: S^{3} \rightarrow B X_{Q}$ misses the cell of $X_{Y}^{D}$. Actually, the map $\xi_{D, C}$ can, by definition, be constructed by only the usual dual composition of $S^{3}$ by $D$. Hence, the map $\xi_{D, C}: S^{3} \rightarrow B X_{Q}$ factors through the rack space $B X$, which implies that the homotopy class $\Xi_{X}(D ; C)$ is derived from $\pi_{3}(B X)$. Therefore, for the study of the invariant, it sufficies to analyze the image of $\left(i_{X}\right)_{*}: \pi_{3}(B X) \rightarrow \pi_{3}\left(B X_{Q}\right)$, where $i_{X}$ is the inclusion $B X \hookrightarrow B X_{Q}$. Let $\pi_{3}^{Q}(B X)$ denote the image $\operatorname{Im}\left(\left(i_{X}\right)_{*}\right)$.

In conclusion, we then define

Definition 2.3 Let $X$ be a finite quandle. We define the group $\pi_{3}^{Q}(B X)$ by the image of the induced map $\pi_{3}(B X) \rightarrow \pi_{3}\left(B X_{Q}\right)$ by the inclusion $B X \hookrightarrow B X_{Q}$. We furthermore define a quandle homotopy invariant of a linked surface $L$ by the following expression:

$$
\Xi_{X}(L)=\sum_{C \in \operatorname{Col}_{X}(D)} \Xi_{X}(D ; C) \in \mathbb{Z}\left[\pi_{3}^{Q}(B X)\right] .
$$


Here $D$ is a broken diagram of $L$, and $\mathrm{Col}_{X}(D)$ denotes the set of $X$-colorings of $D$.

We later study the container $\pi_{3}^{Q}(B X)$ concretely in Sect. 3.1.

\subsection{Reconstrucion of generalized quandle cocycle invariants}

We now reformulate the (generalized) quandle cocycle invariants in $[3,4,6]$ from our quandle homotopy invariant. We first remark that, if $B X^{Y}$ is path-connected, the inclusion $B X^{Y} \hookrightarrow$ $B X_{Q}^{Y}$ induces an isomorphism $\pi_{1}\left(B X^{Y}\right) \cong \pi_{1}\left(B X_{Q}^{Y}\right)$ since $X_{D}^{Y}$ given in (1) has no 1-cell by definition. Given an action of $\pi_{1}\left(B X_{Q}\right)=\operatorname{As}(X)$ on an abelian group $A$, we set a 3cocycle $\psi \in H^{3}\left(B X_{Q} ; A\right)$ with local coefficients. The homology $H_{3}\left(B X_{Q} ; A\right)$ coincides with the quandle homology $H_{3}^{Q}(X ; A)$ introduced in [3, 2 and $\left.\S 7\right]$ (see also Sect. 4.1). Let $\mathfrak{H}: \pi_{3}\left(B X_{Q}\right) \rightarrow H_{3}\left(B X_{Q} ; A\right)$ be the Hurewicz homomorphism with local coefficients. Then the quandle cocycle invariant of $L$ is defined by

$$
\Phi_{\psi}(L)=\sum_{C \in \operatorname{Col}_{X}(D)}\left\langle\psi, \mathfrak{H}\left(\Xi_{X}(D ; C)\right)\right\rangle \in \mathbb{Z}[A]
$$

To summarize, this equality implies that the quandle homotopy invariant is universal among the quandle cocycle invariants.

We give some remarks on the formula (2). It is not difficult to see that the formula (2) coincides with the combinatorial definition of the (generalized) quandle cocycle invariants in $[3, \S 7]$ by the definition of the Hurewicz homomorphism and the construction of the map $\xi_{D, C}$. Therefore the equality (2) means that by knowing a concrete presentation of $\psi$ we can calculate some parts of the quandle homotopy invariant. However, since there are many choices of actions $\pi_{1}(B X) \curvearrowright A$, it is a problem to determine those of of local systems for which the associated quandle cocycle invariants pick out the quandle homotopy invariant. Section 3.1 gives an answer with respect to some Alexander quandles.

Finally, we discuss a local system. Regarding $X$ itself as an $X$-set, the free $A$-module $A\langle X\rangle$ generated by $x \in X$ is a $\pi_{1}(B X)$-module. We then consider a 4-cocycle $\psi \in H^{4}\left(B X_{Q} ; A\right)$ to be a cocycle of $H^{3}\left(B X_{Q} ; A\langle X\rangle\right)$ (see Remark 4.1 for detail); The quandle cocycle invariant of $\psi$ coincides with the shadow cocycle invariant described in [6, §5].

\subsection{Properties of quandle homotopy invariants}

We state some properties of quandle homotopy invariants of linked surfaces, similar to those of classical links in $[23, \S 5]$. For this, we review connected components of a quandle $X$. By definition of quandles, we note that $(\bullet * y): X \rightarrow X$ is bijective for any $y \in X$. Let $\operatorname{Inn}(X)$ denote the subgroup of $\mathfrak{S}_{|X|}$ generated by all the right actions $(\bullet * y)$, called the inner automorphism group of $X$. The connected components of a quandle $X$ are defined by the orbits of the action of $\operatorname{Inn}(X)$ on $X$. For example, let $T_{\ell}$ denote the trivial quandle of order $\ell$ given by the operation $x * y=x$ for $x, y \in T_{\ell}$; Each point in $T_{\ell}$ is its own orbit. Furthermore, a quandle $X$ is said to be connected, if the right action of $\operatorname{Inn}(X)$ on $X$ is transitive. For example, it is known [17, Proposition 1] that an Alexander quandle $X$ is connected if and only if $(1-T)$ is invertible in $X$.

We give a slight reduction of quandle homotopy invariants:

Proposition 2.4 (cf. [23, Lemma 5.5]) Let $X$ be a finite connected quandle, $x$ an element of $X$ and $D$ a link diagram of a linked surface $L$. We fix a sheet of $D$, and denote by $\operatorname{Col}_{X}^{x}(D)$ 
the subset of $\operatorname{Col}_{X}(D)$ such that the sheet is colored by $x \in X$. Then,

$$
\Xi_{X}(L)=|X| \sum_{C \in \operatorname{Col}_{X}^{x}(D)} \Xi_{X}(D ; C) \in \mathbb{Z}\left[\pi_{3}^{Q}(B X)\right] .
$$

Proof For any $y \in X$, We see that the bijection $(\bullet * y): X \rightarrow X$ induces a bijection $\Upsilon_{y}: \operatorname{Col}_{X}^{x}(D) \cong \operatorname{Col}_{X}^{x * y}(D)$ satisfying $\Xi_{X}(D ; C)=\Xi_{X}\left(D ; \Upsilon_{y}(C)\right) \in \pi_{3}^{Q}(B X)$ (see the proof of [14, Proposition 5.2]). Therefore, since $X$ is connected, for any $z \in X$, we have

$$
\sum_{C \in \operatorname{Col}_{X}^{x}(D)} \Xi_{X}(D ; C)=\sum_{C \in \operatorname{Col}_{X}^{z}(D)} \Xi_{X}(D ; C) \in \mathbb{Z}\left[\pi_{3}^{Q}(B X)\right],
$$

which immediately results the required equality (3).

Furthermore, we can show the formulas for the connected sum and the mirror image of the quandle homotopy invariant. We give the formulas without proofs since the proofs are similar to the discussions in $[23, \S 5]$ on the quandle homotopy invariant of classical links.

Proposition 2.5 (cf. [23, Proposition 5.1]) Let X be a connected quandle of finite order. Then, for knotted surfaces $K_{1}$ and $K_{2}$,

$$
\Xi_{X}\left(K_{1} \# K_{2}\right)=\frac{1}{|X|} \Xi_{X}\left(K_{1}\right) \cdot \Xi_{X}\left(K_{2}\right) \in \mathbb{Z}\left[\pi_{3}^{Q}(B X)\right],
$$

where $K_{1} \# K_{2}$ denotes the connected sum of $K_{1}$ and $K_{2}$.

Proposition 2.6 (cf. [23, Proposition 5.7]) Let L be a linked surface. Let $-L^{*}$ denote the mirror image of $L$ with opposite orientation. For any finite quandle $X, \Xi_{X}\left(-L^{*}\right)=\iota\left(\Xi_{X}(L)\right)$, where $\iota$ is the map of $\mathbb{Z}\left[\pi_{3}^{Q}(B X)\right]$ induced by $\iota(x)=x^{-1}$ for any $x \in \pi_{3}^{Q}(B X)$.

The similar formulas to Propositions 2.4, 2.5 hold for the quandle cocycle invariants via the equality (2).

\section{Results about $\pi_{3}^{Q}(B X)$ and $\pi_{2}\left(B X_{Q}\right)$}

In Sect. 2.2, we defined an invariant valued in $\mathbb{Z}\left[\pi_{3}^{Q}(B X)\right]$. We will state our results on the free and torsion parts of the group $\pi_{3}^{Q}(B X)$ in Sect. 3.1. We also compute $\pi_{2}\left(B X_{Q}\right)$ in Sect. 3.2. Here recall from Definition 2.1 that $\pi_{2}\left(B X_{Q}\right)$ denotes the second homotopy group of the quandle space. Meanwhile the container $\pi_{3}^{Q}(B X)$ is the image of the map $\pi_{3}(B X) \rightarrow \pi_{3}\left(B X_{Q}\right)$ induced by the inclusion $B X \hookrightarrow B X_{Q}$ (Definition 2.3).

3.1 Results about $\pi_{3}^{Q}(B X)$

To begin with, we determine the free subgroup of $\pi_{3}^{Q}(B X)$ as follows:

Theorem 3.1 Let $X$ be a finite quandle with $\ell$-connected components (see Sect. 2.4 for the definition). Then $\pi_{3}^{Q}(B X)$ is finitely generated. Furthermore, the rational homotopy groups are determined by the following equalities:

$$
\operatorname{dim}_{\mathbb{Q}}\left(\pi_{2}\left(B X_{Q}\right) \otimes \mathbb{Q}\right)=\frac{\ell^{2}-\ell}{2}, \quad \operatorname{dim}_{\mathbb{Q}}\left(\pi_{3}^{Q}(B X) \otimes \mathbb{Q}\right)=\frac{\ell(\ell-1)(\ell-2)}{3} .
$$

In particular, if $X$ is connected, then $\pi_{3}^{Q}(B X)$ is a finite abelian group. 
The proof will appear in Sect. 4.2. Furthermore we later discuss a topological meaning of the quandle homotopy invariant after tensoring with $\mathbb{Q}$ valued in $\pi_{3}^{Q}(B X) \otimes \mathbb{Q} \cong \mathbb{Q}^{\frac{\ell(\ell-1)(\ell-2)}{3}}$ (Remark 4.2).

Furthermore, we give a corollary:

Corollary 3.2 Let $X$ be a finite connected quandle. Then, for any 3-cocycle $\phi \in H_{Q}^{3}(X ; \mathbb{Z})$ with local coefficients, the quandle cocycle invariant $\Phi_{\phi}(L)$ is trivial. Namely, $\Phi_{\phi}(L) \in$ $\mathbb{Z}\left[0_{\mathbb{Z}}\right]$.

Proof Notice $\pi_{3}^{Q}(B X) \otimes \mathbb{Q} \cong 0$ by Theorem 3.1. Since $\Phi_{\phi}(L)$ is derived from the ring $\mathbb{Z}\left[\pi_{3}^{Q}(B X) \otimes \mathbb{Q}\right]$ by $(2)$, the value of $\Phi_{\phi}(L)$ is trivial.

Remark 3.3 In [3, Tables 3,4 and 5], for a connected quandle of order 3, a non-trivial quandle cocycle invariant $\Phi_{\kappa}(L)$ valued in $\mathbb{Z}\left[\mathbb{Z}^{3}\right]$ was proposed. These values are, however, incorrect, e.g., compare Propositions 2.4 and 2.6 with the tables.

Our next step is to study the torsion subgroup of $\pi_{3}^{Q}(B X)$. However, in general, it is difficult to compute explicitly homotopy groups of spaces. In this paper, we then confine ourselves to $\pi_{3}^{Q}(B X)$ of regular Alexander quandles $X$.

First, we give an estimate of $\pi_{3}(B X)$ by homologies of the rack space $B X$ in homological contexts. The homology of $B X$ is usually called rack homology of $X$ (see Sect. 4.1 for details).

Proposition 3.4 Let $X$ be a regular Alexander quandle of odd order. Let $H_{j}^{R}(X)$ denote the integral homology $H_{j}(B X ; \mathbb{Z})$. Let $Y_{2}$ be a product of Eilenberg-MacLane spaces $K\left(\pi_{2}(B X), 2\right) \times K\left(H_{2}^{R}(X), 1\right)$. Then there exists an exact sequence

$$
H_{4}\left(Y_{2} ; \mathbb{Z}\right) \longrightarrow \pi_{3}(B X) \longrightarrow H_{4}^{R}(X) \longrightarrow H_{3}^{\mathrm{gr}}\left(H_{2}^{R}(X) ; \mathbb{Z}\right) \oplus\left(H_{2}^{R}(X) \otimes \pi_{2}(B X)\right) \rightarrow 0 .
$$

Here $H_{3}^{\mathrm{gr}}\left(H_{2}^{R}(X) ; \mathbb{Z}\right)$ is the third group homology of the abelian group $H_{2}^{R}(X)$.

Proposition 3.4 will be proven by a routine calculation of Postnikov tower in Sect. 5. We see later that $\pi_{2}(B X)$ is determined by $H_{2}^{R}(X)$ and $H_{3}^{R}(X)$ [Theorem 3.9 and (5)]; in conclusion, $\pi_{3}(B X)$ and its quotient $\pi_{3}^{Q}(B X)$ are estimated from upper bounds using the rack homologies $H_{j}^{R}(X)$ with $j \leq 4$, although the exact sequence is not useful to compute $\pi_{3}(B X)$ and $\pi_{3}^{Q}(B X)$ exactly.

However, with an additional assumption, we determine $\pi_{3}^{Q}(B X)$ of regular Alexander quandles $X$ :

Theorem 3.5 Let $X$ be a regular Alexander quandle of odd order. Let $H_{n}^{Q}(X ; \mathbb{Z})$ be the quandle homology with trivial coefficients (see the definition in Sect. 4.1). Assume that the second homology $H_{2}^{Q}(X ; \mathbb{Z})$ vanishes. Then $\pi_{3}(B X) \cong H_{4}^{Q}(X ; \mathbb{Z}) \oplus \mathbb{Z} / 2 \mathbb{Z}$ and $\pi_{3}^{Q}(B X) \cong$ $H_{4}^{Q}(X ; \mathbb{Z})$.

Remark 3.6 There are many Alexander quandles satisfying the assumption. For example, we later show (Lemma A.6) that, if $X$ is of the form $X=\mathbb{F}_{q}[T] /(T-\omega)$ and $\mathbb{F}_{q}$ is an extension of odd degree, then $H_{2}^{Q}(X ; \mathbb{Z}) \cong 0$.

We defer the proof until Sect. 4.2. As a corollary, we now determine $\pi_{3}^{Q}(B X)$ for all Alexander quandles $X$ of prime order:

Corollary 3.7 For an odd prime $p$, we let $X$ be the Alexander quandle of the form $\mathbb{Z}[T] /(p, T-\omega)$. If $\omega \neq-1$, then $\pi_{3}^{Q}(B X) \cong 0$. If $\omega=-1$, then $\pi_{3}^{Q}(B X) \cong \mathbb{Z}_{p}$. 
Proof Mochizuki showed $H_{2}^{Q}(X ; \mathbb{Z}) \cong 0$ [19, Corollary 2.2]. It was shown [24, Corollary 2.3] that if $\omega \neq-1$, then $H_{4}^{Q}(X ; \mathbb{Z}) \cong 0$, and that if $\omega=-1$, then $H_{4}^{Q}(X ; \mathbb{Z}) \cong \mathbb{Z}_{p}$.

Furthermore, we now consider the dihedral quandle, i.e., the case $\omega=-1$. The third cohomology $H_{Q}^{3}\left(X ; \mathbb{F}_{p}\right) \cong \mathbb{F}_{p}$ is shown $[19,20]$, and the generator $\theta_{p} \in H_{Q}^{3}\left(X ; \mathbb{F}_{p}\right)$ is called Mochizuki 3-cocycle. Any quandle cocycle invariant using the dihedral quandle is summarized to assess the quandle cocycle invariant of the Mochizuki 3-cocycle as follows.

Corollary 3.8 Let $X=\mathbb{Z}_{p}[T] /(T+1)$ be the dihedral quandle of order $p$. The group $\pi_{3}^{Q}(B X) \cong \mathbb{Z}_{p}$ is generated by $\Xi_{X}(K ; C)$, where $C$ is an $X$-coloring of the 2-twist spun $(2, p)$-torus knot $K$. Furthermore, any quandle cocycle invariant is equal to a scalar multiple of the quandle cocycle invariant $\Phi_{\theta_{p}}(L) \in \mathbb{Z}\left[\mathbb{F}_{p}\right]$ using the Mochizuki 3-cocycle $\theta_{p} \in$ $H_{Q}^{3}\left(X ; \mathbb{F}_{p}\right)$.

Proof It is known [1, Theorem 6.3] that the quandle cocycle invariant $\Phi_{\theta_{p}}(K)$ of $K$ is nontrivial. Therefore $\pi_{3}^{Q}(B X) \cong \mathbb{Z}_{p}$ is generated by $\Xi_{X}(K ; C)$ for some $X$-coloring $C$, and the $\operatorname{map}\left\langle\theta_{p}, \mathfrak{H}(\bullet)\right\rangle: \pi_{3}^{Q}(B X) \rightarrow \mathbb{F}_{p}$ is an isomorphism. Hence, for any cocycle $\psi \in H_{Q}^{3}(X ; A)$, the quandle cocycle invariant is derived from $\Phi_{\theta_{p}}(L)$ via the inverse map $\left\langle\theta_{p}, \mathfrak{H}(\bullet)\right\rangle^{-1}$ by the formula (2).

\subsection{Computation of the second homotopy groups of quandle spaces $\pi_{2}\left(B X_{Q}\right)$}

Changing the subject, we discuss the quandle homotopy invariant of 1-dimensional links considered in [23]. This invariant is valued in the group ring $\mathbb{Z}\left[\pi_{2}\left(B X_{Q}\right)\right]$ and is universal among the generalized cocycle invariants in $[3, \S 6]$, similar to the equality (2) (see [23, §2] for details). For a finite connected quandle $X$, it is shown [23, Theorem 3.6 and Proposition 3.12] that $\pi_{2}\left(B X_{Q}\right)$ is finite and $\pi_{2}(B X)=\pi_{2}\left(B X_{Q}\right) \oplus \mathbb{Z}$; The author further computed $\pi_{2}\left(B X_{Q}\right)$ for connected quandles of order $\leq 6[23, \S 5]$.

In this paper, we explicitly compute $\pi_{2}\left(B X_{Q}\right)$ of regular Alexander quandles $X$ of odd order in the following theorem, which we will prove in Sect. 5:

Theorem 3.9 Let $X$ be a regular Alexander quandle of finite order.

(i) If $H_{2}^{Q}(X ; \mathbb{Z})$ vanishes, then $\pi_{2}\left(B X_{Q}\right)$ is isomorphic to $H_{3}^{Q}(X ; \mathbb{Z})$.

(ii) If $|X|$ is odd, the following exact sequence splits:

$$
0 \longrightarrow \pi_{2}\left(B X_{Q}\right) \longrightarrow H_{3}^{Q}(X ; \mathbb{Z}) \longrightarrow \Lambda^{2}\left(H_{2}^{Q}(X ; \mathbb{Z})\right) \longrightarrow 0 .
$$

Hence we give a stronger estimate of the torsion part of $\pi_{2}\left(B X_{Q}\right)$ than [23, Theorem 3.6].

Corollary 3.10 Let $X$ be a regular Alexander quandle of odd order. Any element of $\pi_{2}\left(B X_{Q}\right)$ is annihilated by $|X|$.

Proof It is known [24, Corollary 6.2] that $H_{3}^{Q}(X ; \mathbb{Z})$ is annihilated by $|X|$.

We next give two applications. First, we explicitly compute homotopy groups $\pi_{2}\left(B X_{Q}\right)$ for some regular Alexander quandles $X$. If we concretely know $H_{2}^{Q}(X ; \mathbb{Z})$ and $H_{3}^{Q}(X ; \mathbb{Z})$, then Theorem 3.9 enables us to compute $\pi_{2}\left(B X_{Q}\right)$. For example, the computations of all regular Alexander quandles $X$ with $5 \leq|X| \leq 9$ are listed in Table 1 below, where the results of the homologies $H_{2}^{Q}(X), H_{3}^{Q}(X ; \mathbb{Z})$ follow from [17, Table 1]. For more example, in Appendix A we present a computation of $\pi_{2}\left(B X_{Q}\right) \otimes \mathbb{Z}_{p}$ for all Alexander quandles on $\mathbb{F}_{q}$ with $p>2$.

In another application, the result of $\pi_{2}(B X)$ determines a third quandle homology: 
Corollary 3.11 (cf. $[25, \S 3]$ ) For an odd $m$, let $X=\mathbb{Z}[T] /(m, T+1)$ be the dihedral quandle. Then the third quandle homology $H_{3}^{Q}(X ; \mathbb{Z})$ is $\mathbb{Z}_{m}$.

Proof $H_{2}^{Q}(X ; \mathbb{Z}) \cong 0$ is known (see, e.g., [26]). Furthermore, $\pi_{2}(B X) \cong \mathbb{Z}_{m}$ is shown [15, Corollary 5.9]. ${ }^{3}$ Hence, by Theorem 3.9 (i), we have $H_{3}^{Q}(X ; \mathbb{Z}) \cong \mathbb{Z}_{m}$.

Remark 3.12 Mochizuki [21] determined the dimension of $H_{3}^{Q}(X ; \mathbb{Z}) \otimes \mathbb{Z}_{p}$ for any prime p. Our result determines the torsion part of $H_{3}^{Q}(X ; \mathbb{Z})$.

\section{Proof of Theorem 3.1}

Our purpose is to prove Theorem 3.1 in Sect. 4.2 about the rational homotopy groups $\pi_{*}^{Q}(B X) \otimes \mathbb{Q}$. A computation of $\pi_{3}(B X)$ will involve a preliminary analysis of the homology $H_{i}\left(B X^{G} ; \mathbb{Z}\right)$. For this, in Sect. 4.1 , we review the rack homology and some topological monoids.

\subsection{Reviews of rack homology, quandle homology and topological monoid}

We first review the rack complexes introduced by [13] (see also [10]) and the quandle homologies defined in [3]. For a quandle $X$, we denote by $C_{n}^{R}(X)$ the free $\mathbb{Z}[\operatorname{As}(X)]$-module generated by $n$-elements of $X$. Namely, $C_{n}^{R}(X)=\mathbb{Z}[\operatorname{As}(X)]\left\langle X^{n}\right\rangle$. Define a boundary homomorphism $\partial_{n}: C_{n}^{R}(X) \rightarrow C_{n-1}^{R}(X)$, for $n \geq 2$, by

$$
\begin{aligned}
\partial_{n}\left(x_{1}, \ldots, x_{n}\right)= & \sum_{1 \leq i \leq n}(-1)^{i}\left(x_{i}\left(x_{1} * x_{i}, \ldots, x_{i-1} * x_{i}, x_{i+1}, \ldots, x_{n}\right)\right. \\
& \left.-\left(x_{1}, \ldots, x_{i-1}, x_{i+1}, \ldots, x_{n}\right)\right)
\end{aligned}
$$

and $\partial_{1}\left(x_{1}\right)$ to be $x_{1}-1 \in \mathbb{Z}[\operatorname{As}(X)]$. Note that the composite $\partial_{n-1} \circ \partial_{n}$ is zero. Given a left $\mathbb{Z}[\operatorname{As}(X)]$-module $M$, a complex $C_{n}^{R}(X ; M)=M \otimes_{\mathbb{Z}[\operatorname{As}(X)]} C_{n}^{R}(X)$ with $\partial_{n}$ is called the rack complex of $X$ with coefficients $M$. Next, let $C_{n}^{D}(X ; M)$ be a submodule of $C_{n}^{R}(X ; M)$ generated by $n$-tuples $\left(x_{1}, \ldots, x_{n}\right)$ with $x_{i}=x_{i+1}$ for some $i \in\{1, \ldots, n-1\}$ if $n \geq$ 2 ; otherwise, let $C_{1}^{D}(X ; M)=0$. Since $\partial_{n}\left(C_{n}^{D}(X ; M)\right) \subset C_{n-1}^{D}(X ; M)$, we denote the homology by $H_{n}^{D}(X ; \mathbb{Z})$. Furthermore, a complex $\left(C_{*}^{Q}(X ; M), \partial_{*}\right)$ is defined by the quotient $C_{n}^{R}(X ; M) / C_{n}^{D}(X ; M)$. The homology $H_{n}^{Q}(X ; M)$ is called a quandle homology of $X$ with coefficient $M$.

We compare these complexes with the rack spaces. For an $X$-set $Y$, regarding the free module $M=\mathbb{Z}\langle Y\rangle$ as a $\mathbb{Z}[\operatorname{As}(X)]$-module, the complexes $\left(C_{*}^{R}(X ; M), \partial_{*}\right)$ and $\left(C_{*}^{Q}(X ; M), \partial_{*}\right)$ are chain isomorphic to the cellular complexes of $B X^{Y}$ and of $B X_{Q}^{Y}$, respectively. As the simplest case, if $Y$ is a single point, then $\left(C_{*}^{R}(X ; \mathbb{Z}), \partial_{*}\right)$ and $\left(C_{*}^{Q}(X ; \mathbb{Z}), \partial_{*}\right)$ coincide with the rack complex and the quandle complex of $X$ described in [4], respectively.

Remark 4.1 In the special case $Y=X$, we can identify $C_{n+1}^{R}(X ; \mathbb{Z})$ with $C_{n}^{R}(X ; \mathbb{Z}\langle X\rangle)$ from the definitions. Moreover, the identification is a chain map, leading $H_{n+1}^{R}(X ; \mathbb{Z}) \cong$ $H_{n}^{R}(X ; \mathbb{Z}\langle X\rangle) \cong H_{n}\left(B X^{X} ; \mathbb{Z}\right)$ [see [14, Theorem 5.12] for details].

${ }_{3}$ In [15], we dealt with a certain group $\Pi_{2}\left(D_{m}\right)$ instead of $\pi_{2}\left(B X_{Q}\right)$. However, $\pi_{2}\left(B X_{Q}\right) \cong \Pi_{2}\left(D_{m}\right)$ is known. 
We next review basic properties of the complexes. Let $X$ be a finite quandle with $\ell$ connected components and $M=\mathbb{Z}$. According to [17, Theorem 2.2], we have

$$
\begin{array}{r}
H_{1}^{R}(X ; \mathbb{Z}) \cong \mathbb{Z}^{\ell}, \quad H_{2}^{R}(X ; \mathbb{Z}) \cong H_{2}^{Q}(X ; \mathbb{Z}) \oplus \mathbb{Z}^{\ell}, \\
H_{3}^{R}(X ; \mathbb{Z}) \cong H_{3}^{Q}(X ; \mathbb{Z}) \oplus H_{2}^{Q}(X ; \mathbb{Z}) \oplus \mathbb{Z}^{\ell^{2}} .
\end{array}
$$

Furthermore, it is known [5, Lemma 3.2] that

$$
\operatorname{dim}_{\mathbb{Q}}\left(H_{n}^{R}(X ; \mathbb{Z}) \otimes \mathbb{Q}\right)=\ell^{n}, \quad \operatorname{dim}_{\mathbb{Q}}\left(H_{n}^{Q}(X ; \mathbb{Z}) \otimes \mathbb{Q}\right)=\ell \cdot(\ell-1)^{n-1} .
$$

In addition, the following long exact sequence splits (see [17, Theorem 2.1]):

$$
\cdots \rightarrow H_{n+1}^{Q}(X ; \mathbb{Z}) \rightarrow H_{n}^{D}(X ; \mathbb{Z}) \rightarrow H_{n}^{R}(X ; \mathbb{Z}) \rightarrow H_{n}^{Q}(X ; \mathbb{Z}) \rightarrow H_{n-1}^{D}(X ; \mathbb{Z}) \rightarrow \cdots
$$

In particular, we have $H_{n}^{R}(X ; \mathbb{Z}) \cong H_{n}^{Q}(X ; \mathbb{Z}) \oplus H_{n}^{D}(X ; \mathbb{Z})$.

Given a connected quandle $X$ and an $X$-set $Y$, we observe the rack space $B X^{Y}$ as a covering as follows. Note that the collapsing map $Y \rightarrow$ \{pt. $\}$ induces a continuous map $B X^{Y} \rightarrow B X$. It is known (see [13, Theorem 3.7]) that this is a covering; hence, so is the induced map $B X_{Q}^{Y} \rightarrow B X_{Q}$. As a special case, if $Y=G$ is a quotient group of $\operatorname{As}(X)$ (hence $B X^{G}$ is pathconnected), then the two coverings $B X^{G} \rightarrow B X$ and $B X_{Q}^{G} \rightarrow B X_{Q}$ are principal $G$-bundles over $B X$ and $B X_{Q}$, respectively (see [8, Proposition 2.11]). Recalling $\pi_{1}(B X) \cong \operatorname{As}(X)$ from Sect. 2.1, the group $\pi_{1}\left(B X^{G}\right)$ is exactly the kernel of $p_{G}: \operatorname{As}(X) \rightarrow G$

The action of $\pi_{1}\left(B X^{G}\right)$ on the higher homotopy groups $\pi_{*}\left(B X^{G}\right)$ is known to be trivial (see [14, Proposition 5.2] and [8, Proposition 2.16]). Furthermore, the action of $\pi_{1}(B X)$ on the higher homology group $H_{*}\left(B X^{G}\right)$ is also trivial (see [10, Lemma 3.1] or [8, Remark 7]); hence, the action on $H_{*}\left(B X_{Q}^{G}\right)$ is also trivial, since $\pi_{1}(B X)=\pi_{1}\left(B X_{Q}\right)$ and $H_{*}\left(B X_{Q}^{G}\right)$ is a quotient of $H_{*}\left(B X^{G}\right)$ by (7).

Finally, we discuss topological monoids on some rack spaces introduced by Clauwens [8]. Let $G$ be either $\operatorname{As}(X)$ or $\operatorname{Inn}(X)$, and let $G$ act on $X$ canonically. Then, he introduced an operation given by

$$
\begin{aligned}
& \mu:\left(G \times[0,1]^{n} \times X^{n}\right) \times\left(G \times[0,1]^{m} \times X^{m}\right) \rightarrow G \times[0,1]^{n+m} \times X^{n+m}, \\
& \mu\left(\left[g ; t_{1}, \ldots, t_{n}, x_{1}, \ldots, x_{n}\right],\left[h ; t_{1}^{\prime}, \ldots, t_{m}^{\prime}, x_{1}^{\prime}, \ldots, x_{m}^{\prime}\right]\right) \\
& \quad:=\left[g h ; t_{1}, \ldots, t_{n}, t_{1}^{\prime}, \ldots, t_{m}^{\prime}, x_{1} \cdot h, \ldots, x_{n} \cdot h, x_{1}^{\prime}, \ldots, x_{m}^{\prime}\right],
\end{aligned}
$$

where $n, m \in \mathbb{Z}_{>0}$; this further gives rise to a topological monoid structure on $B X^{G}$ (see $[8, \S 2.5])$. In particular, $\pi_{1}\left(B X^{G}\right)$ is an abelian group. Since $B X^{G}$ is a so-called nilpotent space, we often deal with the localization of $B X^{G}$ at a prime $p$.

\subsection{Proof of Theorem 3.1}

Proof Consider $G=\operatorname{Inn}(X)$, which is a finite group. Since the rack space $B X^{G}$ is a topological monoid and is a path-connected CW-complex of finite type, $B X^{G}$ is homotopic to a (based) loop space of some simply connected CW-complex $W$ of finite type, that is, $B X^{G} \simeq \Omega W$ as an $H$-space (see [22, Theorem 1.5 and §2] for details). In particular, it is without saying that $\pi_{*}\left(B X^{G}\right)$ is finitely generated; hence, so is the quotient $\pi_{3}^{Q}(B X)$.

Let us focus on rational homologies of $B X^{G}$ and $B X_{Q}^{G}$. Recall that the projections $p_{Q}^{G}$ : $B X_{Q}^{G} \rightarrow B X_{Q}$ and $p_{G}: B X^{G} \rightarrow B X$ are coverings of degree $|G|$, and that the actions of 
$\pi_{1}(B X)$ on $H_{*}\left(B X_{Q}^{G}\right)$ and on $H_{*}\left(B X^{G}\right)$ are trivial. It then follows from the transfer maps that $p_{Q}^{G}$ and $p_{G}$ induce two isomorphisms:

$$
\left(p_{G}\right)_{*}: H_{n}\left(B X^{G} ; \mathbb{Q}\right) \cong H_{n}(B X ; \mathbb{Q}), \quad\left(p_{Q}^{G}\right)_{*}: H_{n}\left(B X_{Q}^{G} ; \mathbb{Q}\right) \cong H_{n}\left(B X_{Q} ; \mathbb{Q}\right) .
$$

One discusses $\pi_{*}\left(B X^{G}\right) \otimes \mathbb{Q}$. Since $B X^{G} \simeq \Omega W$ mentioned above, we recall the known following formula (see $[12, \S 33(\mathrm{c})])$ :

$$
\sum_{i \geq 0} \operatorname{dim}\left(H^{i}\left(B X^{G} ; \mathbb{Q}\right)\right) t^{i}=\prod_{i \geq 0} \frac{\left(1+t^{2 i+1}\right)^{r_{2 i+1}}}{\left(1-t^{2 i}\right)^{r_{2 i}}} \in \mathbb{Z}[[t]],
$$

where we put $r_{i}=\operatorname{dim}\left(\pi_{i}\left(B X^{G}\right) \otimes \mathbb{Q}\right)$. By (6) and (8), we then easily have $r_{2}=\left(\ell^{2}+\ell\right) / 2$ and $r_{3}=\left(\ell^{3}-\ell\right) / 3$. Since $\pi_{2}(B X) \cong \pi_{2}\left(B X_{Q}\right) \oplus \mathbb{Z}^{\ell}$ is known (see [23, Proposition 3.12]), we conclude $\operatorname{dim}\left(\pi_{2}\left(B X_{Q}\right) \otimes \mathbb{Q}\right)=\left(\ell^{2}-\ell\right) / 2$.

We next calculate $\pi_{3}^{Q}(B X) \otimes \mathbb{Q}$. For this, we equip $H^{*}\left(B X^{G} ; \mathbb{Q}\right)$ with a Hopf algebra arising from the monoid structure, and denote by $P^{*}\left(B X^{G}\right)$ the set of primitive elements of $H^{*}\left(B X^{G} ; \mathbb{Q}\right)$. By the Milnor-Moore theorem (see, e.g., [12, Theorem 21.5]), $\pi_{*}\left(B X^{G}\right) \otimes$ $\mathbb{Q} \cong P^{*}\left(B X^{G}\right)$ and the dual Hurewicz map $(\mathfrak{H})^{*} \otimes \mathbb{Q}: H^{*}\left(B X^{G} ; \mathbb{Q}\right) \rightarrow \operatorname{Hom}\left(\pi_{*}\left(B X^{G}\right), \mathbb{Q}\right)$ coincides with the projection $H^{*}\left(B X^{G} ; \mathbb{Q}\right) \rightarrow P^{*}\left(B X^{G}\right)$.

We now explain the isomorphism (10) below. By the isomorphisms (8) and the splitting in (7), we notice that the induced map $\left(i_{X}\right)_{*}: H_{*}\left(B X^{G} ; \mathbb{Q}\right) \rightarrow H_{*}\left(B X_{Q}^{G} ; \mathbb{Q}\right)$ is a split surjection, where $i_{X}$ is the inclusion $B X^{G} \hookrightarrow B X_{Q}^{G}$. Furthermore, we consider the long exact sequences of cohomology and homotopy groups of the pair $\left(B X_{Q}^{G}, B X^{G}\right)$, and have the following commutative diagram:

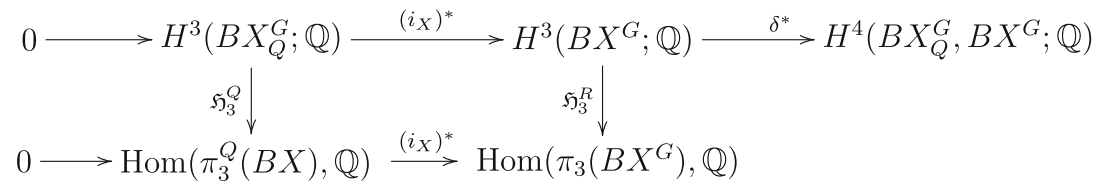

Here the top and bottom sequences are exact, and the vertical arrows are the Hurewicz homomorphisms. By the Milnor-Moore theorem again, we obtain

$$
\pi_{3}^{Q}(B X) \otimes \mathbb{Q} \cong P^{3}\left(B X^{G}\right) \cap \operatorname{Ker}\left(\delta^{*}: H^{3}\left(B X^{G} ; \mathbb{Q}\right) \rightarrow H^{4}\left(B X_{Q}^{G}, B X^{G} ; \mathbb{Q}\right)\right) .
$$

We will calculate the right hand side $P^{3}\left(B X^{G}\right) \cap \operatorname{Ker}\left(\delta^{*}\right)$ using the complexes in Sect. 4.1. Inspired by the monoid structure on $B X^{G}$, we consider a map $\mu:\left(G \times X^{n}\right) \times\left(G \times X^{m}\right) \rightarrow$ $G \times X^{n+m}$ given by

$$
\mu\left(\left(g ; x_{1}, \ldots, x_{n}\right),\left(h ; x_{1}^{\prime}, \ldots, x_{m}^{\prime}\right)\right):=\left(g h ; x_{1} \cdot h, \ldots, x_{n} \cdot h, x_{1}^{\prime}, \ldots, x_{m}^{\prime}\right) .
$$

This provides $H_{D}^{*}(X ; \mathbb{Q}[G])$ and $H_{R}^{*}(X ; \mathbb{Q}[G])$ with Hopf algebra structures. ${ }^{4}$ Further, note that the inclusion $i_{D}^{G}: C_{*}^{D}(X ; \mathbb{Q}[G]) \rightarrow C_{*}^{R}(X ; \mathbb{Q}[G])$ induces a Hopf algebra homomorphism $\left(i_{D}^{G}\right)^{*}: H_{R}^{*}(X ; \mathbb{Q}[G]) \rightarrow H_{D}^{*}(X ; \mathbb{Q}[G])$. Let us identify the above map $\delta^{*}$ with the induced map $\left(i_{D}^{G}\right)^{*}$ by definitions. By (7) we then notice that

$$
\begin{aligned}
& \operatorname{Ker}\left(\delta^{*}\right) \cap H^{3}\left(B X^{G} ; \mathbb{Q}\right) \cong \operatorname{Ker}\left(\left(i_{D}^{G}\right)^{*}\right) \cap H_{R}^{3}(X ; \mathbb{Q}[G]) \cong H_{Q}^{3}(X ; \mathbb{Q}) \cong \mathbb{Q}^{\ell(\ell-1)^{2}}, \\
& \operatorname{Ker}\left(\left(i_{D}^{G}\right)^{*}\right) \cap P^{1}\left(B X^{G}\right) \cong H_{R}^{1}(X ; \mathbb{Q}[G]) \cong \mathbb{Q}^{\ell} .
\end{aligned}
$$

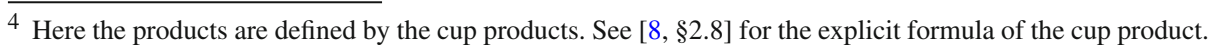


In conclusion, the dimension of $P^{3}\left(B X^{G}\right) \cap \operatorname{Ker}\left(\delta^{*}\right)$ in (10) is equal to

$$
\begin{aligned}
\operatorname{dim} & \left(H_{Q}^{3}(X ; \mathbb{Q}[G])\right)-\operatorname{dim}\left(\Lambda^{3}\left(P^{1}\left(B X^{G}\right)\right)\right)-\operatorname{dim}\left(P^{1}\left(B X^{G}\right) \otimes P^{2}\left(B X^{G}\right)\right) \\
& =\ell(\ell-1)^{2}-\frac{\ell(\ell-1)(\ell-2)}{6}-\ell \frac{\left(\ell^{2}+\ell\right)}{2}=\frac{\ell(\ell-1)(\ell-2)}{3} .
\end{aligned}
$$

Remark 4.2 We roughly explain a topological meaning of the quandle homotopy invariant after tensoring with $\mathbb{Q}$ as follows. To illustrate, we put a collapsing map $X \rightarrow T_{\ell}$ on each connected components, where $T_{\ell}$ is the trivial quandle of order $\ell$. We see that this map induces $\pi_{3}^{Q}(B X) \otimes \mathbb{Q} \cong \pi_{3}^{Q}\left(B T_{\ell}\right) \otimes \mathbb{Q}$ by a functoriality parallel to the previous proof; we may assume $X=T_{\ell}$. Then we can obtain all the 3-cocycles of $H_{R}^{3}\left(T_{\ell} ; \mathbb{Q}\right) \cong \mathbb{Q}^{\ell^{3}}$, since the coboundary maps $\delta_{n}$ are zero by definition. Since $\pi_{3}^{Q}\left(B T_{\ell}\right) \otimes \mathbb{Q}$ is derived from the primitive elements of $H_{3}^{R}\left(T_{\ell} ; \mathbb{Q}\right)$ by the Milnor-Moore theorem, we can evaluate elements of $\pi_{3}^{Q}\left(B T_{\ell}\right) \otimes \mathbb{Q}$ by the 3 -cocycles.

On the other hand, we consider the bordism group $L_{\ell}^{2}$ with $\ell$-connected components (see $[7, \S 1]$ for the definition). An isomorphism $L_{\ell}^{2} \otimes \mathbb{Q} \cong \mathbb{Q}^{\frac{\ell(\ell-1)(\ell-2)}{3}}$ is known; further it is shown [7] that $L_{\ell}^{2} \otimes \mathbb{Q}$ is generated by "Hopf 2-links". Recall the map $\Xi_{X}(D ; \bullet): \operatorname{Col}_{X}(D) \rightarrow$ $\pi_{3}\left(B X_{Q}\right)$ with $X=T_{\ell}$ in Sect. 2.2. By running over all $T_{\ell}$-colorings of all broken diagrams, the maps give rise to a homomorphism $L_{\ell}^{2} \otimes \mathbb{Q} \rightarrow \pi_{3}^{Q}(B X) \otimes \mathbb{Q}$. Since we easily compute the rational quandle homotopy invariant of the Hopf 2-links with $X=T_{\ell}$ by pairing with the previous 3-cocycles, the homomorphism turns to be an isomorphism $L_{\ell}^{2} \otimes \mathbb{Q} \cong \pi_{3}^{Q}(B X) \otimes \mathbb{Q}$.

\section{Proofs of Theorems 3.5 and 3.9}

Our purpose in this section is to prove Theorem 3.5 in Sect. 5.1 and Theorem 3.9 in Sect. 5.2.

We now outline the proof of Theorem 3.5. To see this, we first aim to study $\pi_{3}(B X)$ since $\pi_{3}^{Q}(B X)$ is a quotient of $\pi_{3}(B X)$. Let $\widetilde{B X}$ be the universal covering of $B X$. By the monoid structure of $\widetilde{B X}$ explained in Sect. 4.1, $\widetilde{B X}$ is a loop space of a 2-connected CWcomplex. Thanks to the fact [2] that the second $k$-invariant of $\widetilde{B X}$ is annihilated by 2, we will show $\pi_{3}(B X)=\pi_{3}(\widetilde{B X}) \cong H_{3}(\widetilde{B X} ; \mathbb{Z}) \oplus \pi_{3}\left(\Omega S^{2}\right)$ by a routine argument of the Postnikov tower. In Sect. 5.1, we see $H_{3}(\widetilde{B X} ; \mathbb{Z}) \cong H_{4}^{Q}(X ; \mathbb{Z})$ using techniques of quandle homologies (see (11)). Finally, we show that $H_{4}^{Q}(X ; \mathbb{Z})$ does not vanish in $\pi_{3}^{Q}(B X)$, leading $\pi_{3}^{Q}(B X) \cong H_{4}^{Q}(X ; \mathbb{Z})$ as stated in Theorem 3.5. Theorem 3.9 is also proven in a similar way.

To carry out the outline, we here prepare two propositions, which are proven in Sect. 5.3.

Proposition 5.1 Let $X$ be a regular Alexander quandle of finite order. Let $G=\operatorname{Inn}(X)$. Then $H_{2}\left(B X^{G} ; \mathbb{Z}\right) \cong H_{3}^{Q}(X ; \mathbb{Z}) \oplus H_{2}^{Q}(X ; \mathbb{Z}) \oplus \mathbb{Z}$. Furthermore, if $H_{2}^{Q}(X ; \mathbb{Z}) \cong 0$, then

(i) $H_{3}\left(B X^{G} ; \mathbb{Z}\right) \cong H_{4}^{Q}(X ; \mathbb{Z}) \oplus H_{3}^{Q}(X ; \mathbb{Z}) \oplus \mathbb{Z}$.

(ii) $H_{3}\left(B X_{Q}^{G} ; \mathbb{Z}\right) \cong H_{4}^{Q}(X ; \mathbb{Z}) \oplus H_{3}^{Q}(X ; \mathbb{Z})$.

(iii) The map $H_{3}\left(B X^{G} ; \mathbb{Z}\right) \rightarrow H_{3}\left(B X_{Q}^{G} ; \mathbb{Z}\right)$ induced by the inclusion $B X^{G} \hookrightarrow B X_{Q}^{G}$ coincides with the projection in the sense of the above presentations ( $i)$ and (ii).

Proposition 5.2 Let $X$ be a regular Alexander quandle of finite order. Let $A s(X) \rightarrow \operatorname{Inn}(X)$ be the epimorphism in Sect. 4.1. Then the kernel is isomorphic to $\pi_{1}\left(B X^{G}\right) \cong H_{2}^{R}(X ; \mathbb{Z}) \cong$ $\mathbb{Z} \oplus H_{2}^{Q}(X ; \mathbb{Z})$. 
We furthermore require an elementary lemma:

Lemma 5.3 Let $\mathcal{M}$ be a topological monoid with the binary operation $\mu$. If $\mathcal{M}$ is a pathconnected $C W$-complex and $\pi_{1}(\mathcal{M}) \cong \mathbb{Z}$, then $\mathcal{M}$ is homotopic to $S^{1} \times \widetilde{\mathcal{M}}$. Here $\widetilde{\mathcal{M}}$ is the universal covering of $\mathcal{M}$.

Proof Choose a representative $f: S^{1} \rightarrow \mathcal{M}$ of a generator of $\pi_{1}(\mathcal{M})$. Let $g: \widetilde{\mathcal{M}} \rightarrow \mathcal{M}$ denote the universal covering map. The composite map $\mu \circ(f \times g): S^{1} \times \widetilde{\mathcal{M}} \rightarrow \mathcal{M}$ turns out to give a weak homotopy equivalence as desired.

\subsection{Proof of Theorem 3.5}

Proof From now on, $X$ is assumed to be a regular Alexander quandle with $H_{2}^{Q}(X ; \mathbb{Z}) \cong 0$. By Proposition 5.2, the kernel of the epimorphism $\operatorname{As}(X) \rightarrow \operatorname{Inn}(X)$ is entirely $\mathbb{Z}$. Hence, setting $G=\operatorname{Inn}(X)$, we have $B X^{G} \simeq S^{1} \times \widetilde{B X}$ by Lemma 5.3 ; thus it follows from Proposition 5.1 and the Kunneth formula that

$$
H_{2}(\widetilde{B X} ; \mathbb{Z}) \simeq \mathbb{Z} \oplus H_{3}^{Q}(X ; \mathbb{Z}), \quad H_{3}(\widetilde{B X} ; \mathbb{Z}) \simeq H_{4}^{Q}(X ; \mathbb{Z}) .
$$

Let us calculate the localizations of $\pi_{3}(\widetilde{B X})$ at every prime $p$. Since $\widetilde{B X}$ is also a topological monoid, the $\widetilde{B X}$ is a loop space. Let $\widetilde{B X}_{i}$ denote the $i$-th stage of Postnikov tower. It immediately follows from [2, Theorem 3.2] that the third $k$-invariant $k^{4} \in$ $H^{4}\left(K\left(\pi_{2}(\widetilde{B X}), 2\right) ; \pi_{3}(\widetilde{B X})\right)$ satisfies $2 k^{4}=0$ (see also [30, Proposition 3]). Hence, since the order $|X|$ is odd, the localization of $\widetilde{B X}_{3}$ at a prime $p>2$ is reduced to be

$$
\left(\widetilde{B X}_{(p)}\right)_{3} \simeq K\left(\pi_{2}(B X)_{(p)}, 2\right) \times K\left(\pi_{3}(B X)_{(p)}, 3\right) .
$$

Hence, the Hurewicz map $\mathfrak{H}_{(p)}$ passes to an isomorphism $\pi_{3}(B X)_{(p)} \cong H_{3}(\widetilde{B X} ; \mathbb{Z})_{(p)}$, since $H_{3}\left(K\left(\pi_{2}(B X), 2\right) ; \mathbb{Z}\right)$ vanishes (see $\left.\left[18, \S 8^{b i s}\right]\right)$. As a consequence, combining with (11) immediately gives $\pi_{3}(B X)_{(p)} \cong H_{3}(\widetilde{B X} ; \mathbb{Z})_{(p)} \cong H_{4}^{Q}(X ; \mathbb{Z})_{(p)}$ with $p>2$.

We consider the last case $p=2$. It is known [14, Theorem 5.12] that $B T_{1}$ is homotopic to $\Omega S^{2}$, where $T_{1}$ is the trivial quandle of order 1 . Let $X \rightarrow T_{1}$ be the collapsing map. This induces a topological monoid homomorphism $f: B X^{G} \rightarrow \Omega S^{2}$. By Lemma 5.7 below, the torsion subgroup of $H_{*}\left(B X^{G} ; \mathbb{Z}\right)$ is annihilated by $|X|$; therefore, $\left(f_{(2)}\right)_{*}: H_{*}\left(B X^{G} ; \mathbb{Z}\right)_{(2)} \rightarrow H_{*}\left(\Omega S^{2} ; \mathbb{Z}\right)_{(2)}\left(\cong \mathbb{Z}_{(2)}[\zeta]\right)$ is isomorphic, where $\zeta$ is a generator of $H_{1}\left(\Omega S^{2} ; \mathbb{Z}\right) \cong \mathbb{Z}$. Hence, by the Whitehead theorem, the cellular map $f$ gives rise to a homotopy equivalence $f_{(2)}: B X_{(2)}^{G} \rightarrow \Omega S_{(2)}^{2}$. In particular, $\pi_{3}\left(B X_{(2)}^{G}\right) \cong \pi_{3}\left(\Omega S^{2}\right)_{(2)} \cong \pi_{4}\left(S^{2}\right)_{(2)} \cong \mathbb{Z}_{2}$. Furthermore, by Theorem 3.1 and (6), we note $\pi_{3}(B X)_{(0)} \cong H_{4}^{Q}(X ; \mathbb{Z})_{(0)} \cong 0$. In summary, we conclude $\pi_{3}(B X) \cong H_{4}^{Q}(X ; \mathbb{Z}) \oplus \mathbb{Z}_{2}$ as required.

Next, we discuss $\pi_{3}^{Q}(B X)$. We now show an isomorphism $\pi_{3}\left(B X^{G}\right)_{(p)} \rightarrow \pi_{3}\left(B X_{Q}^{G}\right)_{(p)}$ with $p>2$. Considering the covering $g: \widetilde{B X} \rightarrow B X^{G}$ and the inclusion $i_{X}: B X^{G} \hookrightarrow$ $B X_{Q}^{G}$, we have a commutative diagram

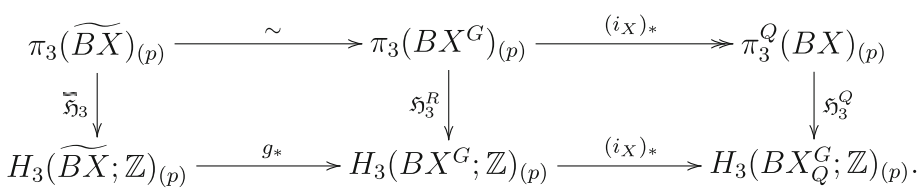

For $p>2$, we remark that the localization of the Hurewicz map $\widetilde{\mathfrak{H}}_{3}$ is an isomorphism by (12). By Lemma 5.3, the map $g_{*}$ is injective; hence, so is $\mathfrak{H}_{3}^{R}$. We note that, by Proposition 5.1, 
the above map $\left(i_{X}\right)_{*}: H_{3}\left(B X^{G} ; \mathbb{Z}\right) \rightarrow H_{3}\left(B X_{Q}^{G} ; \mathbb{Z}\right)$ is isomorphic. Therefore, by diagram chasing, the bottom map $\left(i_{X}\right)_{*}: \pi_{3}\left(B X^{G}\right)_{(p)} \rightarrow \pi_{3}\left(B X_{Q}^{G}\right)_{(p)}$ is the isomorphism.

To complete the proof, it suffices to show that the direct summand $\mathbb{Z}_{2}$ of $\pi_{3}(B X)$ is sent to the zero via $\left(i_{X}\right)_{*}: \pi_{3}(B X) \rightarrow \pi_{3}\left(B X_{Q}\right)$. By the above discussion of the direct summand $\mathbb{Z}_{2}$, we may assume $X=T_{1}$. It is known [7,28] that $\pi_{3}\left(B T_{1}\right) \cong \mathbb{Z}_{2}$ is isomorphic to the framed link bordism group $\left(\cong \mathbb{Z}_{2}\right)$, and that the generator of the bordism group is represented by an embedding of a pair of unknotted tori (see [28, Example 1.12] for details). Hence, from the definition of $B X_{Q}$, for any $T_{1}$-coloring $C$ of the tori, the cellular map $\xi_{D, C}$ in Sect. 2.2 is bounded by a 3-cell of the subspace $X_{Y}^{D}$ in Sect. 2.1. Namely, the homotopy class $\left[\xi_{D, C}\right]$ vanishes in $\pi_{3}\left(B X_{Q}\right)$, which completes the proof.

Remark 5.4 In a similar manner, we can calculate the forth homotopy groups $\pi_{4}(B X)$ of regular Alexander quandles $X$ of odd order with $H_{2}^{Q}(X ; \mathbb{Z})=0$ as follows. In fact, it is shown [2, Theorem 4.6] that the forth $k$-invariant of $B X$ is annihilated by 16 ; hence the forth stage $(\widetilde{B X})_{4}$ localized at $p>2$ is presented by

$$
\left(\widetilde{B X}_{(p)}\right)_{4} \simeq K\left(\pi_{2}(B X)_{(p)}, 2\right) \times K\left(\pi_{3}(B X)_{(p)}, 3\right) \times K\left(\pi_{4}(B X)_{(p)}, 4\right) .
$$

About the case $p=2$, it follows from the proceeding map $f_{(2)}$ that $\pi_{4}(B X)_{(2)}=$ $\pi_{4}\left(\Omega S^{2}\right)_{(2)}=\pi_{5}\left(S^{2}\right)_{(2)}=\mathbb{Z}_{2}$. Hence, noting $H_{4}\left((\widetilde{B X})_{4} ; \mathbb{Z}\right) \cong H_{4}(\widetilde{B X} ; \mathbb{Z})$, Lemma 5.3 concludes that the group $\pi_{4}(B X)$ can be calculated from $\pi_{2}(B X), \pi_{3}(B X)$ and $H_{4}\left(B X^{G} ; \mathbb{Z}\right)$.

For instance, let us compute $\pi_{4}(B X)$ in the dihedral case $X=\mathbb{Z}[T] /(p, T+1)$ with $p>2$. Recall that $\pi_{3}(B X)=\mathbb{Z}_{2 p}$ from Corollary 3.7, and that $\pi_{2}(B X)=\mathbb{Z} \oplus \mathbb{Z}_{p}$ from Theorem 3.9. In addition, $H_{4}\left(B X^{G} ; \mathbb{Z}\right)=\mathbb{Z} \oplus \mathbb{Z}_{p}^{3}$ is known $[8, \S 1.4]$, which implies $H_{4}(\widetilde{B X} ; \mathbb{Z})=\mathbb{Z} \oplus \mathbb{Z}_{p}^{2}$ by Lemma 5.3. Therefore, by (13) we conclude $\pi_{4}(B X)=\mathbb{Z}_{2}$ (cf. Corollary 3.8).

\subsection{Proofs of Theorem 3.9 and Proposition 3.4}

We let $X$ be a regular Alexander quandle without the assumption $H_{2}^{Q}(X ; \mathbb{Z})=0$, and $|X|$ be odd. We fix a notation $G=\operatorname{Inn}(X)$. To prove Theorem 3.9, we use the Postnikov tower of the rack space $B X$ (see, e.g., [18, Lemma $\left.8^{\text {bis }} .27\right]$ with $n=2$ ), which is an exact sequence

$$
H_{3}^{\mathrm{gr}}\left(\pi_{1}\left(B X^{G}\right) ; \mathbb{Z}\right) \longrightarrow \pi_{2}\left(B X^{G}\right) \stackrel{\mathfrak{H}}{\longrightarrow} H_{2}\left(B X^{G} ; \mathbb{Z}\right) \longrightarrow H_{2}^{\mathrm{gr}}\left(\pi_{1}\left(B X^{G}\right) ; \mathbb{Z}\right) \longrightarrow 0 .(14)
$$

Here $H_{*}^{\mathrm{gr}}\left(\pi_{1}\left(B X^{G}\right) ; \mathbb{Z}\right)$ is the group homology of $\pi_{1}\left(B X^{G}\right)$.

Proof of Theorem 3.9 (i) By assumption of $H_{2}^{Q}(X ; \mathbb{Z})=0$, we have $\pi_{1}\left(B X^{G}\right) \cong \mathbb{Z}$ by Proposition 5.2. Noting $H_{r}^{\mathrm{gr}}(\mathbb{Z} ; \mathbb{Z}) \cong 0$ for $r \geq 2$, the Hurewicz map $\mathfrak{H}$ in (14) is isomorphic. Recall $H_{2}\left(B X^{G} ; \mathbb{Z}\right) \cong H_{3}^{Q}(X ; \mathbb{Z}) \oplus H_{2}^{Q}(X ; \mathbb{Z}) \oplus \mathbb{Z}$ by Proposition 5.1. Since $\pi_{2}(B X) \cong \mathbb{Z} \oplus \pi_{2}\left(B X_{Q}\right)$, we have $\pi_{2}(B X) \cong H_{3}^{Q}(X ; \mathbb{Z})$ as required.

(ii) To obtain the required sequence (4), we will show that the map $\mathfrak{H}$ in (14) is a split injection. Recall that $B X^{G}$ is a path-connected loop space. It immediately follows from [2, Theorem 3.2] that the second $k$-invariant $k^{3} \in H_{\mathrm{gr}}^{3}\left(\pi_{1}\left(B X^{G}\right) ; \pi_{2}\left(B X^{G}\right)\right)$ satisfies $2 k^{3}=$ 0 . By Proposition 5.2, $\pi_{1}\left(B X^{G}\right) \cong H_{2}^{R}(X ; \mathbb{Z})$, which implies that the group cohomology $H_{\mathrm{gr}}^{3}\left(\pi_{1}\left(B X^{G}\right) ; \pi_{2}\left(B X^{G}\right)\right)$ is annihilated by $|X|$. Hence, the second $k$-invariant is zero. That is, the second stage of the Postnikov tower is homotopic to $K\left(\pi_{1}\left(B X^{G}\right) ; 1\right) \times$ $K\left(\pi_{2}\left(B X^{G}\right) ; 2\right)$, and the map $\mathfrak{H}$ is a split injection (cf. [2, pp 3]).

We now calculate each terms in the sequence (14) above. Using Proposition 5.2, the second group homology in (14) is expressed as

$$
H_{2}^{\mathrm{gr}}\left(\pi_{1}\left(B X^{G}\right) ; \mathbb{Z}\right) \cong H_{2}^{\mathrm{gr}}\left(\mathbb{Z} \oplus H_{2}^{Q}(X ; \mathbb{Z}) ; \mathbb{Z}\right) \cong H_{2}^{Q}(X ; \mathbb{Z}) \oplus\left(H_{2}^{Q}(X ; \mathbb{Z}) \wedge_{\mathbb{Z}} H_{2}^{Q}(X ; \mathbb{Z})\right) \text {. }
$$


Since $H_{2}\left(B X^{G} ; \mathbb{Z}\right)=\mathbb{Z} \oplus H_{2}^{Q}(X ; \mathbb{Z}) \oplus H_{3}^{Q}(X ; \mathbb{Z})$ by Proposition 5.1, the sequence (14) is then rewritten in

$$
\begin{aligned}
0 \rightarrow \pi_{2}(B X) \stackrel{\mathfrak{H}}{\longrightarrow} \mathbb{Z} \oplus H_{2}^{Q}(X ; \mathbb{Z}) & \oplus H_{3}^{Q}(X ; \mathbb{Z}) \longrightarrow H_{2}^{Q}(X ; \mathbb{Z}) \\
& \oplus\left(H_{2}^{Q}(X ; \mathbb{Z}) \wedge_{\mathbb{Z}} H_{2}^{Q}(X ; \mathbb{Z})\right) \rightarrow 0 .
\end{aligned}
$$

Since $\pi_{2}(B X) \cong \mathbb{Z} \oplus \pi_{2}\left(B X_{Q}\right)$ and the restriction of $\mathfrak{H}$ to the $\mathbb{Z}$-part is known to be isomorphic (see [23, Proposition 3.12]), we immediately obtain the required sequence (4).

Remark 5.5 As is seen in the proof, the Hurewicz homomorphism $\mathfrak{H}$ in the sequence (14) is injective. Hence, via $\mathfrak{H}$, any element of $\pi_{2}\left(B X_{Q}\right)$ can be evaluated by some cocycles $\psi \in H_{Q}^{2}(X ; A) \oplus H_{Q}^{3}(X ; A)$ with trivial coefficients. In conclusion, there exist $\psi_{1}, \ldots, \psi_{m} \in$ $H_{Q}^{2}(X ; A) \oplus H_{Q}^{3}(X ; A)$ such that any quandle cocycle invariant constructed from $X$ is a linear sum of the quandle cocycle invariants associated with $\psi_{1}, \ldots, \psi_{m}$.

Furthermore, by a similar discussion, we now show Proposition 3.4 as follows:

Proof of Proposition 3.4 Let $Y_{i}$ denote the $i$-th stage of the Postnikov tower of $B X^{G}$. We recall the following exact sequence in [18, Lemma $\left.8^{\text {bis }} .27\right]$.

$$
H_{4}\left(Y_{2} ; \mathbb{Z}\right) \longrightarrow \pi_{3}\left(B X^{G}\right) \stackrel{\mathfrak{H}}{\longrightarrow} H_{3}\left(B X^{G} ; \mathbb{Z}\right) \longrightarrow H_{3}\left(Y_{2} ; \mathbb{Z}\right) \longrightarrow 0 .
$$

Let us calculate the each terms in (15). By Lemma 5.9, the third homology $H_{3}\left(B X^{G} ; \mathbb{Z}\right)$ is isomorphic to the rack homology $H_{4}^{R}(X ; \mathbb{Z})$. Moreover, it follow from the previous proof of Theorem 3.9 that $Y_{2}$ is homotopic to $K\left(\pi_{1}\left(B X^{G}\right), 1\right) \times K\left(\pi_{2}\left(B X^{G}\right), 2\right)$. Furthermore, recall the isomorphism $H_{2}^{R}(X ; \mathbb{Z}) \cong \pi_{1}\left(B X^{G}\right)$ from Proposition 5.2. Therefore, we easily obtain the required sequence stated in Proposition 3.4 from the sequence (15) and the Kunneth formula.

\subsection{Proofs of Propositions 5.1 and 5.2}

In Sects. 5.3 and 5.4, $X$ is assumed to be a finite connected Alexander quandle. We fix a notation $G=\operatorname{Inn}(X)$. To show Propositions 5.1 and 5.2, we first study $\operatorname{Inn}(X)$ as follows.

Lemma 5.6 Let $e \in \mathbb{Z}_{>0}$ be the minimal number satisfying $\left(T^{e}-1\right) X=0$. Then $\operatorname{Inn}(X) \cong$ $\mathbb{Z} / e \mathbb{Z} \ltimes X$. Here $\mathbb{Z} / e \mathbb{Z}$ acts on $X$ by the multiplication by $T$.

Proof For $(\varepsilon, x) \in \mathbb{Z} / e \mathbb{Z} \ltimes X$, we put an element of $\operatorname{Map}(X, X)$ sending $y \in X$ to $T^{\varepsilon} y+$ $(1-T) x \in X$. This then gives rise to an epimorphism $\mathbb{Z} / e \mathbb{Z} \ltimes X \rightarrow \operatorname{Inn}(X)\left(\subset \mathfrak{S}_{|X|}\right)$. We claim that this is injective. Actually, if $(\varepsilon, x) \in \mathbb{Z} / e \mathbb{Z} \ltimes X$ satisfies $y=T^{\varepsilon} y+(1-T) x$ for any $y \in X$, then we easily obtain $(\varepsilon, x)=(0,0)$.

As a result, we see that the surjection $\operatorname{As}(X) \rightarrow \operatorname{Inn}(X)$ in Sect. 4.1 coincides with a homomorphism $\operatorname{As}(X) \rightarrow \mathbb{Z} / e \mathbb{Z} \ltimes X$ sending $x$ to $(1, x)$. Recalling the isomorphism $H_{n}\left(B X^{G} ; \mathbb{Z}\right) \cong H_{n}^{R}(X ; \mathbb{Z}[\operatorname{Inn}(X)])$ mentioned in Sect. 4.1, we also observe another lemma:

Lemma 5.7 Let $X$ be a finite connected Alexander quandle. Let $M$ be $\mathbb{Z}[\operatorname{Inn}(X)]$. Then the torsion subgroup of $H_{*}^{R}(X ; M)=H_{*}\left(B X^{G} ; \mathbb{Z}\right)$ is annihilated by $|X|$.

We defer its proof until Sect. 5.4. We next study a relation between $H_{n}^{Q}(X ; M)$ and $H_{n}\left(B X_{Q}^{G} ; \mathbb{Z}\right)$. To see this, we review a complex $C_{n}^{L}(X)$ introduced in $[17, \S 2]$. This $C_{n}^{L}(X)$ is defined to be the subcomplex of $C_{n}^{D}(X)$ generated by $n$-tuples $\left(x_{1}, \ldots, x_{n}\right) \in X^{n}$ with 
$x_{i}=x_{i+1}$ for some $2 \leq i<n$. As is shown [17, Lemma 9], there is an isomorphism of chain complexes:

$$
C_{*}^{D}(X ; \mathbb{Z}) \cong C_{*-1}^{Q}(X ; \mathbb{Z}) \oplus C_{*}^{L}(X ; \mathbb{Z}),
$$

where the direct summand $C_{*}^{L}(X ; \mathbb{Z})$ is obtained from the inclusion $C_{*}^{L}(X ; \mathbb{Z}) \hookrightarrow C_{*}^{D}(X ; \mathbb{Z})$.

Lemma 5.8 If $H_{2}^{Q}(X ; \mathbb{Z})=0$, then $H_{4}^{L}(X ; \mathbb{Z}) \cong \mathbb{Z}$.

We later prove this in Sect. 5.4. Meanwhile, we consider the complex $C_{*}^{R}(X ; \mathbb{Z}\langle X\rangle)$ in Remark 4.1. Under the identification $C_{n}^{R}(X ; \mathbb{Z}\langle X\rangle) \cong C_{n+1}^{R}(X ; \mathbb{Z})$, as this restriction, we have a chain isomorphism $C_{n}^{D}(X ; \mathbb{Z}\langle X\rangle) \cong C_{n+1}^{L}(X ; \mathbb{Z})$. In conclusion, by (7) and (16), we thus have

$$
H_{n}\left(B X_{Q}^{X} ; \mathbb{Z}\right) \cong H_{n}^{Q}(X ; \mathbb{Z}\langle X\rangle) \cong H_{n+1}^{Q}(X ; \mathbb{Z}) \oplus H_{n}^{Q}(X ; \mathbb{Z}) .
$$

Lemma 5.9 Let $X$ be a regular Alexander quandle of finite order. Then $H_{n}\left(B X^{G} ; \mathbb{Z}\right) \cong$ $H_{n+1}^{R}(X ; \mathbb{Z})$ for $n \geq 1$. Furthermore, $H_{n}\left(B X_{Q}^{G} ; \mathbb{Z}\right) \cong H_{n+1}^{Q}(X ; \mathbb{Z}) \oplus H_{n}^{Q}(X ; \mathbb{Z})$.

Proof We first show $H_{n}\left(B X^{G} ; \mathbb{Z}\right) \cong H_{n+1}^{R}(X ; \mathbb{Z})$. Let us regard $X$ as an $X$-set (see Sect. 2.1). We consider the two maps $\operatorname{Inn}(X) \rightarrow X$ and $X \rightarrow\{$ pt. $\}$. They then give rise to two coverings $B X^{G} \rightarrow B X^{X}$ and $B X^{X} \rightarrow B X$ (cf. [8, §4.1]). Furthermore, note that the covering $B X^{G} \rightarrow B X^{X}$ is of degree $e$ by Lemma 5.6. Hence the transfer map yields an isomorphism $H_{n}\left(B X^{G} ; \mathbb{Z}\right)_{(p)} \cong H_{n}\left(B X^{X} ; \mathbb{Z}\right)_{(p)}$ where the prime $p$ divides $|X|$ (see [8, Propositions 4.2]). Furthermore, it is evident that $H_{n}\left(B X^{G} ; \mathbb{Z}\right)_{(0)} \cong H_{n}(B X ; \mathbb{Z})_{(0)} \cong \mathbb{Q}$ by (6). It is shown [24, Theorem 6.3] that the torsion subgroup of $H_{n}(B X ; \mathbb{Z})$ is annihilated by $|X|$. Combining with Lemma 5.7 , we have $H_{n}\left(B X^{G} ; \mathbb{Z}\right) \cong H_{n}\left(B X^{X} ; \mathbb{Z}\right) \cong H_{n+1}(B X ; \mathbb{Z}) \cong H_{n+1}^{R}(X ; \mathbb{Z})$ as required.

To prove the latter part, similarly, we can see $H_{*}\left(B X_{Q}^{G} ; \mathbb{Z}\right) \cong H_{*}\left(B X_{Q}^{X} ; \mathbb{Z}\right)$. Therefore, we have $H_{n}\left(B X_{Q}^{G} ; \mathbb{Z}\right) \cong H_{n+1}^{Q}(X ; \mathbb{Z}) \oplus H_{n}^{Q}(X ; \mathbb{Z})$ by $(17)$, which completes the proof.

Using the lemmas above, we now prove Propositions 5.1 and 5.2.

Proof of Proposition 5.1 The desired isomorphism $H_{2}\left(B X^{G} ; \mathbb{Z}\right) \cong H_{3}^{Q}(X ; \mathbb{Z}) \oplus H_{2}^{Q}(X ; \mathbb{Z})$ $\oplus \mathbb{Z}$ immediately follows from Lemma 5.9 and (7). Similarly, (ii) follows from the lemma.

To prove (i), using (7) and (16), Lemma 5.9 results in

$$
H_{3}\left(B X^{G} ; \mathbb{Z}\right) \cong H_{4}^{R}(X ; \mathbb{Z}) \cong H_{4}^{Q}(X ; \mathbb{Z}) \oplus H_{3}^{Q}(X ; \mathbb{Z}) \oplus H_{4}^{L}(X ; \mathbb{Z}) .
$$

Therefore, the required isomorphism (i) is obtained from Lemma 5.8. Finally, the proof of (iii) readily follows from the sequence (7) and the isomorphism (17).

Proof of Proposition 5.2 Recall that the kernel of the epimorphism $\operatorname{As}(X) \rightarrow \operatorname{Inn}(X)=G$ is $\pi_{1}\left(B X^{G}\right)$ and abelian (see Sect. 4.1). Hence, $\pi_{1}\left(B X^{G}\right) \cong H_{1}\left(B X^{G} ; \mathbb{Z}\right) \cong H_{2}^{R}(X ; \mathbb{Z})$ by Lemma 5.9.

\subsection{Proofs of Lemmas 5.8 and 5.7}

Before proving Lemma 5.8, we remark the condition $H_{2}^{Q}(X ; \mathbb{Z}) \cong 0$. By (5), we have $H_{2}^{R}(X ; \mathbb{Z}) \cong \mathbb{Z}$. It is known $[5,17]$ that, for any $x \in X$, the 2-cycle of the form $(x, x) \in$ $C_{2}^{R}(X ; \mathbb{Z})$ is a generator of $H_{2}^{R}(X ; \mathbb{Z}) \cong \mathbb{Z}$. Namely, any 2-cycle of $C_{2}^{R}(X ; \mathbb{Z})$ is homologous to $\alpha(x, x)$ for some $\alpha \in \mathbb{Z}$. 
Proof of Lemma 5.8 Consider a 4-cycle $\sigma \in C_{4}^{L}(X ; \mathbb{Z})$, i.e., $\partial_{4}(\sigma)=0$. It is enough to show that the cycle $\sigma$ is homologous to a sum of $(y, y, y, y)$ s for some $y \in X$. Let us expand $\sigma$ as $\sum_{i} \alpha_{i}\left(a_{i}, b_{i}, b_{i}, c_{i}\right)+\sum_{j} \beta_{j}\left(d_{j}, e_{j}, f_{j}, f_{j}\right)$ for some $a_{i}, b_{i}, c_{i}, d_{j}, e_{j}, f_{j} \in X$ and $\alpha_{i}, \beta_{j} \in \mathbb{Z}$, where $e_{j} \neq f_{j}$.

We now show the equality (19) below. For any $g_{i} \in X$, we note that

$$
\begin{aligned}
\partial_{5}\left(a_{i}, g_{i}, b_{i}, b_{i}, c_{i}\right)= & \left(a_{i}, b_{i}, b_{i}, c_{i}\right)-\left(a_{i} * g_{i}, b_{i}, b_{i}, c_{i}\right)-\left(a_{i}, g_{i}, b_{i}, b_{i}\right) \\
& +\left(a_{i} * c_{i}, g_{i} * c_{i}, b_{i} * c_{i}, b_{i} * c_{i}\right) .
\end{aligned}
$$

Putting $g_{i}=\left(b_{i}-T a_{i}\right) /(1-T)$, we notice $a_{i} * g_{i}=b_{i}$. Hence, for any $i$, we may assume $a_{i}=b_{i}$. The condition $\partial_{4}(\sigma)=0$ is thus formulated by

$$
\begin{aligned}
0= & \left(\sum_{i} \alpha_{i}\left(a_{i}, a_{i}, a_{i}\right)-\alpha_{i}\left(a_{i} * c_{i}, a_{i} * c_{i}, a_{i} * c_{i}\right)\right) \\
& +\sum_{j} \beta_{j}\left(d_{j}, f_{j}, f_{j}\right)-\beta_{j}\left(d_{j} * e_{j}, f_{j}, f_{j}\right) .
\end{aligned}
$$

One deals with the letter term $\sum_{j} \beta_{j}\left(d_{j}, e_{j}, f_{j}, f_{j}\right)$. We fix $\phi \in X$. Therefore $C_{1}^{R}(X ; \mathbb{Z}) \ni$ $0=\sum_{j} \beta_{j}\left(d_{j}-d_{j} * e_{j}\right)=\partial_{2}\left(\sum_{j} \beta_{j}\left(d_{j}, e_{j}\right)\right)$, where $j$ runs satisfying $f_{j}=\phi$. Since $H_{2}^{R}(X ; \mathbb{Z}) \cong \mathbb{Z}$, the sum $\sum_{j} \beta_{j}\left(d_{j}, e_{j}\right)$ is homologous to $\gamma_{\phi}(\phi, \phi)$ for some $\gamma_{\phi} \in \mathbb{Z}$. Notice

$$
\begin{aligned}
\partial_{5}\left(a_{j}, b_{j}, c_{j}, \phi, \phi\right)= & \left(a_{j}, c_{j}, \phi, \phi\right)-\left(a_{j} * b_{j}, c_{j}, \phi, \phi\right)-\left(a_{j}, b_{j}, \phi, \phi\right) \\
& +\left(a_{j} * c_{j}, b_{j} * c_{j}, \phi, \phi\right),
\end{aligned}
$$

that is, $\partial_{5}\left(a_{j}, b_{j}, c_{j}, \phi, \phi\right)=\partial_{3}\left(a_{j}, b_{j}, c_{j}\right) \otimes(\phi, \phi)$. We therefore conclude that the sum $\sum_{j} \beta_{j}\left(d_{j}, e_{j}, f_{j}, f_{j}\right)$ is homologous to $\sum_{\phi \in X} \gamma_{\phi}(\phi, \phi, \phi, \phi)$.

On the other hand, let us discuss the former term $\sum_{i} \alpha_{i}\left(a_{i}, a_{i}, a_{i}, c_{i}\right)$. By (19), we have $C_{1}^{R}(X ; \mathbb{Z}) \ni 0=\sum_{i} \alpha_{i}\left(a_{i}-a_{i} * c_{i}\right)=\partial_{2}\left(\sum_{i} \alpha_{i}\left(a_{i}, c_{i}\right)\right)$. Since $H_{2}^{R}(X ; \mathbb{Z}) \cong \mathbb{Z}$, the sum $\sum_{i} \alpha_{i}\left(a_{i}, c_{i}\right)$ is homologous to $\alpha(y, y)$ for some $\alpha \in \mathbb{Z}$ and $y \in X$. By definition, we note

$$
\begin{aligned}
\partial_{5}(a, a, a, d, f)= & (a, a, a, f)-(a * d, a * d, a * d, f)-(a, a, a, f) \\
& +(a * f, a * f, a * f, d * f), \\
\partial_{3}(a, d, f)= & (a, b, f)-(a * d, f)-(a, f)+(a * f, d * f) .
\end{aligned}
$$

In conclusion, the sum $\sum_{i} \alpha_{i}\left(a_{i}, a_{i}, a_{i}, c_{i}\right)$ is homologous to $\alpha(y, y, y, y)$ as desired.

Finally, we will prove Lemma 5.7 as follows. For this, it is convenient to change another "coordinate system" of the complex $C_{n}^{R}(X ; \mathbb{Z}[\operatorname{Inn}(X)])$ such as $[20, \S 2.1 .3]$. We denote elements of $\mathbb{Z} / e \mathbb{Z}$ by $\varepsilon$. We then define $C_{n}^{R_{U}}(X)$ to be the free $\mathbb{Z}$-module generated by elements $\left(\varepsilon, U_{0} ; U_{1}, \ldots, U_{n}\right)$ of $\mathbb{Z} / e \mathbb{Z} \times X \times X^{n}$, and the boundary map by

$$
\begin{aligned}
& \partial_{n}\left(\varepsilon, U_{0} ; U_{1}, \ldots, U_{n}\right) \\
& \quad=\sum_{0 \leq i \leq n-1}(-1)^{i}\left(\left(\varepsilon+1, T U_{0} ; T U_{1}, \ldots, T U_{i-1}, T U_{i}+U_{i+1}, U_{i+2}, \ldots, U_{n}\right)\right. \\
& \left.\quad-\left(\varepsilon, U_{0} ; U_{1}, \ldots, U_{i-1}, U_{i}+U_{i+1}, U_{i+2}, \ldots, U_{n}\right)\right) .
\end{aligned}
$$

We can see $\partial_{n-1} \circ \partial_{n}=0$. Furthermore let us consider a bijection given by

$$
\begin{aligned}
\mathbb{Z} / e \mathbb{Z} \times X^{n+1} \ni\left(\varepsilon, x_{0} ; x_{1}, \ldots, x_{n}\right) & \mapsto\left(\varepsilon, x_{0}-x_{1} ; x_{1}-x_{2}, \ldots, x_{n-1}-x_{n}, x_{n}\right) \\
& \in \mathbb{Z} / e \mathbb{Z} \times X^{n+1} .
\end{aligned}
$$


The bijection yields a chain isomorphism from $C_{n}^{R}(X ; \mathbb{Z}[\operatorname{Inn}(X)])$ to $C_{n}^{R_{U}}(X)$, where we use the identification $\operatorname{Inn}(X) \cong \mathbb{Z} / e \mathbb{Z} \ltimes X$ by Lemma 5.6.

Proof of Lemma 5.7 The proof is analogous to [24, Theorem 6.1]. We set a chain homomorphism $\mathcal{Z}: C_{n}^{R_{U}}(X) \rightarrow C_{n}^{R_{U}}(X)$ defined by $\mathcal{Z}\left(\epsilon, U_{0} ; U_{1}, \ldots, U_{n}\right):=|X| \cdot(\epsilon, 0 ; 0, \ldots, 0)$. Furthermore, we define two homomorphisms $D_{n, 0}^{j}: C_{n}^{R_{U}}(X) \rightarrow C_{n+1}^{R_{U}}(X)$ and $D_{n,+}^{j}$ : $C_{n}^{R_{U}}(X) \rightarrow C_{n+1}^{R_{U}}(X)$ by

$D_{n, 0}^{j}\left(\varepsilon, U_{0} ; U_{1}, \ldots, U_{n}\right)=\sum_{y \in X}\left(\varepsilon, 0 ; 0, \ldots, 0, y, U_{j}-y, U_{j+1}, \ldots, U_{n}\right) \quad$ for $0 \leq j \leq n$, $D_{n,+}^{j}\left(\varepsilon, U_{0} ; U_{1}, \ldots, U_{n}\right)= \begin{cases}\sum_{y \in X}\left(\varepsilon, 0 ; 0, \ldots, 0, y,-y, U_{j+1}, \ldots, U_{n}\right) & \text { for } 0 \leq j<n, \\ \sum_{y \in X}(\varepsilon, 0 ; 0, \ldots, 0, y,-y) & \text { for } j=n .\end{cases}$ In addition, we set $D_{n, 0}^{n+1}=D_{n,+}^{n+1}=0$. Then, by direct calculation, we can verify the equality $\sum_{0 \leq j \leq n}(-1)^{j}\left(\partial_{n+1}\left(D_{n, 0}^{j}+D_{n,+}^{j}\right)+\left(D_{n-1,0}^{j}+D_{n-1,+}^{j}\right) \partial_{n}\right)=(-1)^{n}\left(|X| \cdot \mathrm{id}_{C_{n}^{R_{U}(X)}}-\mathcal{Z}\right)$.

Then, we have the induced map $|X| \cdot \mathrm{id}_{H_{n}^{R_{U}}(X)}=(\mathcal{Z})_{*}: H_{n}^{R_{U}}(X) \rightarrow H_{n}^{R_{U}}(X)$. Therefore, for the proof, it suffices to show that any cycle of the form $\sum_{\varepsilon} a_{\varepsilon}(\varepsilon, 0 ; 0, \ldots, 0)$ is contained in the free subgroup of $C_{n}^{R_{U}}(X)$, where $a_{\varepsilon} \in \mathbb{Z}$. Indeed, the augmentation map $C_{n}^{R_{U}}(X) \rightarrow \mathbb{Z}$ is an $n$-cocycle and sends any $(\varepsilon, 0 ; 0, \ldots, 0)$ to 1 .

Acknowledgments The author is grateful to Takuro Mochizuki for helpful communications on quandle cocycles and Remark A.2. He expresses his gratitude to Syunji Moriya for valuable conversations on rational homotopy theory. He is grateful to J. Scott Carter, Inasa Nakamura, Masahico Saito and Kokoro Tanaka for useful discussions on Remark 3.3 and the paper [3]. He sincerely thanks the referee for many suggestions which have improved the exposition

Open Access This article is distributed under the terms of the Creative Commons Attribution License which permits any use, distribution, and reproduction in any medium, provided the original author(s) and the source are credited.

\section{Appendix A: $\pi_{2}\left(B X_{Q}\right) \otimes \mathbb{Z}_{p}$ of Alexander quandles $X$ on $\mathbb{F}_{q}$}

This appendix computes $\pi_{2}\left(B X_{Q}\right) \otimes \mathbb{Z}_{p}$ for Alexander quandles of the forms $X=$ $\mathbb{F}_{q}[T] /(T-\omega)$ (see Appendix A.2). For this, in Appendix A.1, we review some results in $[19,20]$ and observe the betti number $b_{i}^{Q}=\operatorname{dim}_{\mathbb{F}_{p}}\left(H_{i}^{Q}(X ; \mathbb{Z}) \otimes \mathbb{Z}_{p}\right)$.

\section{A.1 Review and some remarks on Mochizuki's cocycles}

Mochizuki explicitly determined the second and third quandle cohomology groups $[19,20]$. To see this, we first recall from [20, Theorem 2.2] that

$$
\begin{aligned}
\operatorname{dim}_{\mathbb{F}_{q}}\left(H_{Q}^{2}\left(X ; \mathbb{F}_{q}\right)\right) & =\#\left\{(i, j) \in \mathbb{Z}^{2} \mid 1 \leq p^{i}<p^{j}<q, \omega^{p^{i}+p^{j}}=1\right\} \\
& =\sum_{0<i<h: \omega^{p^{i}+1}=1} h-i,
\end{aligned}
$$


where $q=p^{h}$. In particular, one notices that $H_{Q}^{2}\left(X ; \mathbb{F}_{q}\right)$ vanishes if and only if $\omega^{p^{i}+1} \neq 1$ for any $i<h$, that is, the order of $\omega$ is not divisible by $p^{i}+1$ for any $i<h$.

Next, we discuss the third quandle cohomology $H_{Q}^{3}\left(X ; \mathbb{F}_{q}\right)$, which was computed in [20]. However, the statement of the main theorem [20, Theorem 2.11] contained some minor mistakes. Then we will state a corrected one. Recall the following polynomials over $\mathbb{F}_{q}$ in $[20, \S 2.2]$ :

$$
\begin{aligned}
F(a, b, c) & :=U_{0}^{a} \cdot U_{1}^{b} \cdot U_{2}^{c}, \\
\chi\left(U_{j}, U_{j+1}\right) & :=\sum_{1 \leq i \leq p-1}(-1)^{i-1} i^{-1} U_{j}^{p-i} U_{j+1}^{i}, \\
E_{0}(a \cdot p, b) & :=\left(\chi\left(\omega U_{0}, U_{1}\right)-\chi\left(U_{0}, U_{1}\right)\right)^{a} \cdot U_{2}^{b}, \\
E_{1}(a, b \cdot p) & :=U_{0}^{a} \cdot\left(\chi\left(U_{1}, U_{2}\right)-\chi\left(\omega^{-1} \cdot U_{1}, U_{2}\right)\right)^{b} .
\end{aligned}
$$

Here for $\left(x_{1}, x_{2}, x_{3}\right) \in X^{3}$, we put $U_{0}=x_{1}-x_{2}, U_{1}=x_{2}-x_{3}$ and $U_{2}=x_{3}$, similar to (20). Then these polynomials are regarded as functions of $C_{3}^{Q}\left(X ; \mathbb{F}_{q}\right)$. Furthermore, we let $\mathcal{Q}(q)$ be the set of quadruples $\left(q_{1}, q_{2}, q_{3}, q_{4}\right)$ satisfying the following conditions:

- $q_{2} \leq q_{3}, q_{1}<q_{3}, q_{2}<q_{4}$, and $\omega^{q_{1}+q_{3}}=\omega^{q_{2}+q_{4}}=1$. Here if $p=2$, we omit $q_{2}=q_{3}$.

- One of the following holds:

- Case $1 \omega^{q_{1}+q_{2}}=1$

- Case $2 \omega^{q_{1}+q_{2}} \neq 1$, and $q_{3}>q_{4}$.

- Case $3(p \neq 2), \omega^{q_{1}+q_{2}} \neq 1$, and $q_{3}=q_{4}$.

- Case $4(p \neq 2), \omega^{q_{1}+q_{2}} \neq 1, q_{2} \leq q_{1}<q_{3}<q_{4}, \omega^{q_{1}}=\omega^{q_{2}}$.

- Case $5(p=2), \omega^{q_{1}+q_{2}} \neq 1, q_{2}<q_{1}<q_{3}<q_{4}, \omega^{q_{1}}=\omega^{q_{2}}$.

For such a quadruple $\left(q_{1}, q_{2}, q_{3}, q_{4}\right) \in \mathcal{Q}(q)$, Mochizuki introduced a polynomical denoted by $\Gamma\left(q_{1}, q_{2}, q_{3}, q_{4}\right)$ (see [20, $\left.\S 2.2 .2\right]$ for the definitions). We state [20, Theorem 2.11] as

Theorem A.1 ([20]) The third quandle cohomology $H_{Q}^{3}\left(X ; \mathbb{F}_{q}\right)$ is spanned by the following set $I_{q, \omega}$ composed of non-trivial 3-cocycles. Here $q_{i}$ means a power of the prime $p$ with $q_{i}<q$.

$$
\begin{aligned}
I_{q, \omega}:= & \left\{F\left(q_{1}, q_{2}, q_{3}\right) \mid \omega^{q_{1}+q_{2}+q_{3}}=1, q_{1}<q_{2}<q_{3}\right\} \\
& \cup\left\{F\left(q_{1}, q_{2}, 0\right) \mid \omega^{q_{1}+q_{2}}=1, q_{1}<q_{2}\right\} \\
& \cup\left\{E_{0}\left(p \cdot q_{1}, q_{2}\right) \mid \omega^{p \cdot q_{1}+q_{2}}=1, q_{1}<q_{2}\right\} \\
& \cup\left\{E_{1}\left(q_{1}, q_{2} \cdot p\right) \mid \omega^{q_{1}+p \cdot q_{2}}=1, q_{1} \leq q_{2}\right\} \\
& \cup\left\{\Gamma\left(q_{1}, q_{2}, q_{3}, q_{4}\right) \mid\left(q_{1}, q_{2}, q_{3}, q_{4}\right) \in \mathcal{Q}(q)\right\} .
\end{aligned}
$$

Remark A.2 In the original statement, the set $I_{q, \omega}$ contained certain 3-cocycles denoted by " $\Psi\left(a, q_{1}\right)$ ". However, we easily see that these 3-cocycles $\Psi\left(a, q_{1}\right)$ are cohomologous to zero (see $[20, \S 2.2 .1]$ ). This minor error results from the mistake " $B_{d-p^{s}}^{2(s)}(q)$ " in the proof of [20, Lemma 3.16] instead of " $B_{d-p^{s}}^{2}(q)$ ".

Remark A.3 By the universal coefficient theorem, we have $\left|I_{q, \omega}\right|=b_{2}^{Q}+b_{3}^{Q}$. 
A. 2 Computations of $\pi_{2}\left(B X_{Q}\right) \otimes \mathbb{Z}_{p}$

We now compute $\pi_{2}\left(B X_{Q}\right) \otimes \mathbb{Z}_{p}$ in the cases of $\omega=-1$, of $q=p^{2 h+1}$, and of $q=p^{2}$. In respect to a regular Alexander quandle $X$ of odd order, the following formula is useful:

$$
\operatorname{dim}_{\mathbb{F}_{p}}\left(\pi_{2}\left(B X_{Q}\right) \otimes \mathbb{Z}_{p}\right)=b_{3}^{Q}-\frac{b_{2}^{Q}\left(b_{2}^{Q}-1\right)}{2},
$$

where $b_{n}^{Q}$ is the $\operatorname{dimension} \operatorname{dim}_{\mathbb{F}_{p}}\left(H_{n}^{Q}(X ; \mathbb{Z}) \otimes \mathbb{Z}_{p}\right)$ with a prime $p>2$. This formula is immediately obtained from Theorem 3.9.

\section{A.2.1 The case of $\omega=-1$}

When $\omega=-1$ and $p$ is odd, let us compute $\pi_{2}\left(B X_{Q}\right) \otimes \mathbb{Z}_{p}$.

Corollary A.4 Let $X$ be the product h-copies of the dihedral quandle $D_{p}$. Then

$$
\operatorname{dim}_{\mathbb{F}_{p}}\left(\pi_{2}\left(B X_{Q}\right) \otimes \mathbb{Z}_{p}\right)=\frac{h^{2}\left(h^{2}+11\right)}{12} .
$$

Proof We can verify that the order of the set $\mathcal{Q}(q)$ defined in Appendix A.1 is $|\mathcal{Q}(q)|=$ $h(h-1)(h+1)(5 h-6) / 24$. Hence, Theorem A. 1 means $\left|I_{q, w}\right|=h\left(5 h^{3}-6 h^{2}+31 h-6\right) / 24$. By $(21)$, notice $b_{2}^{Q}=h(h-1) / 2$. By (23), we thus conclude $\operatorname{dim}_{\mathbb{F}_{p}}\left(\pi_{2}\left(B X_{Q}\right) \otimes \mathbb{F}_{p}\right)=$ $h^{2}\left(h^{2}+11\right) / 12$.

Remark A.5 We notice that the dimension is a quadratic function with respect to $h$. In particular, the second homotopy groups do not preserve the direct products of quandles.

\section{A.2.2 Field extensions $\mathbb{F}_{q}$ of odd degrees with $\omega \neq-1$}

We next calculate $\operatorname{dim}\left(\pi_{2}(B X) \otimes \mathbb{F}_{p}\right)$, when $X=\mathbb{F}_{q}$ is an extension of odd degree and $\omega \neq-1$. To see this, we first show

Lemma A.6 Let $\omega \neq \pm 1$ and $q=p^{2 h+1}$ (possibly $p=2$ ). Then $H_{2}^{Q}(X ; \mathbb{Z}) \cong 0$.

Proof Since $H_{2}^{Q}(X ; \mathbb{Z})$ is shown to be annihilated by $q$ (see [24, Corollary 6.2]), we now show $H_{Q}^{2}\left(X ; \mathbb{F}_{q}\right) \cong 0$ for the proof of $H_{2}^{Q}(X ; \mathbb{Z}) \cong 0$. To this end, it suffices to show $\omega^{p^{i}+1} \neq 1$ for any $i \leq 2 h$ by (21). A key is that, if $p>2$, then $\left(p^{2 m}+1\right) / 2$ and $\left(p^{2 n-1}+1\right) / 2$ are relatively prime for any $n, m \in \mathbb{Z}$; This is easily verified by induction on $n+m$. We now assume $\omega^{p^{i}+1}=1$ for some $i \leq 2 h$. Then $\omega^{p^{2 h-i+1}+1}=\left(\omega^{p^{i}+1} \omega^{p^{2 h+1}-1}\right)^{-p^{i}}=1$. This means that $p^{i}+1$ and $p^{2 h-i+1}+1$ have a common divisor except for 2 , which contradicts the key; Hence $H_{Q}^{2}\left(X ; \mathbb{F}_{q}\right) \cong 0$ in the sequel. A similar discussion holds for the case $p=2$.

Therefore, combing Lemma A.6 with Theorem A.1 immediately concludes

Corollary A.7 If $\mathbb{F}_{q}$ is of odd degree and $\omega \neq-1$, then the dimension $\operatorname{dim}\left(\pi_{2}(B X) \otimes \mathbb{F}_{p}\right)$ is equal to the order $\left|\left\{\left(q_{1}, q_{2}, q_{3}\right) \mid \omega^{q_{1}+q_{2}+q_{3}}=1, q_{1}<q_{2}<q_{3}<q\right\}\right|$.

Example A.8 Let $q=p$. If $\omega \neq-1$, then the second and third quandle cohomologies vanish; hence $\pi_{2}\left(B X_{Q}\right) \cong 0$.

Example A.9 For example, we let $q=p^{3}$. One notices that, when $\omega^{1+p+p^{2}} \neq 1, H_{Q}^{3}\left(X ; \mathbb{F}_{q}\right)$ vanishes; hence, $\pi_{2}\left(B X_{Q}\right) \cong 0$. On the other hand, if $\omega^{1+p+p^{2}}=1$, then $I_{q, \omega}$ consists of the polynomial $F\left(1, p, p^{2}\right)$. Hence, $\pi_{2}\left(B X_{Q}\right) \otimes \mathbb{Z}_{p} \cong \mathbb{Z}_{p}$ 


\section{A.2.3 The case of $q=p^{2}$ with $\omega \neq-1$}

We here compute $\pi_{2}\left(B X_{Q}\right)$ of extension fields $X$ over $\mathbb{F}_{p}$ of degree 2 . Without $\omega=-1$, the dimensions of $\pi_{2}\left(B X_{Q}\right) \otimes \mathbb{Z}_{p}$ are determined by the following three cases.

Example A.10 We consider the case of $\omega^{1+p} \neq 1$. Then we have $b_{2}^{Q}=0$ by (21). Furthermore, by Theorem A.1, we obtain $b_{3}^{Q}=0$. Therefore, $\pi_{2}\left(B X_{Q}\right) \cong 0$.

Example A.11 We consider the case where $\omega \neq-1, \omega^{1+p}=1$ and $p \neq 2$. Then the equalities (21) mean $b_{2}^{Q}=1$. Furthermore, by Theorem A.1, the third cohomology $H_{Q}^{3}\left(X ; \mathbb{F}_{q}\right)$ is spanned by

$$
I_{p^{2}, \omega}=\left\{F(1, p, 0), E_{1}(1, p), E_{1}\left(p, p^{2}\right), \Gamma(1, p, 1, p)\right\} .
$$

Hence $b_{3}^{Q}=3$. Therefore we have $\operatorname{dim}_{\mathbb{F}_{p}}\left(\pi_{2}\left(B X_{Q}\right) \otimes \mathbb{Z}_{p}\right)=3$ by the formula (23).

More generally, for given a quandle of the form $X=\mathbb{F}_{q}[T] /(T-\omega)$ with $q>p^{2}$, we can compute $\operatorname{dim}\left(\pi_{2}\left(B X_{Q}\right) \otimes \mathbb{Z}_{p}\right)$ in a similar manner.

\section{References}

1. Asami, S., Satoh, S.: An infinite family of non-invertible surfaces in 4-space. Bull. Lond. Math. Soc. 37, 285-296 (2005)

2. Arlettaz, D., Pointet-Tischler, N.: Postnikov invariants of H-spaces. Fund. Math. 161(1-2), 17-35 (1999)

3. Carter, J.S., Elhamdadi, J.S., Graña, M., Saito, M.: Cocycle knot invariants from quandle modules and generalized quandle homology. Osaka J. Math. 42, 499-541 (2005)

4. Carter, J.S., Jelsovsky, D., Kamada, S., Langford, L., Saito, M.: Quandle cohomology and state-sum invariants of knotted curves and surfaces. Trans. Am. Math. Soc. 355, 3947-3989 (2003)

5. Carter, J.S., Jelsovsky, D., Kamada, S., Saito, M.: Quandle homology groups, their Betti numbers, and virtual knots. J. Pure Appl. Algebra 157, 135-155 (2001)

6. Carter, J.S., Kamada, S., Saito, M.: Geometric interpretations of quandle homology. J. Knot Theory Ramifications 10, 345-386 (2001)

7. Carter, J.S., Kamada, S., Saito, M., Satoh, S.: A theorem of Sanderson on link Bordisms in dimension 4. Algebraic Geom. Topol. 1, 299-310 (2001)

8. Clauwens, F.J.-B.J.: The algebra of rack and quandle cohomology. J. Knot Theory Ramifications 11, 1487-1535 (2011)

9. Carter, J.S., Saito, M.: Canceling branch points on projections of surfaces in 4-space. Proc. Am. Math. Soc. 116, 229-237 (1992)

10. Etingof, P., Graña, M.: On rack cohomology. J. Pure Appl. Algebra 177, 49-59 (2003)

11. Eisermann, M.: Knot colouring polynomials. Pac. J. Math. 231, 305-336 (2007)

12. Félix, Y., Halperin, S., Thomas, J.C.: Rational homotopy theory. Graduate Texts in Mathematics, vol. 205. Springer, New York (2001)

13. Fenn, R., Rourke, C., Sanderson, B.: Trunks and classifying spaces. Appl. Categ. Struct. 3, 321-356 (1995)

14. Fenn, R., Rourke, C., Sanderson, B.: The rack space. Trans. Am. Math. Soc. 359, 701-740 (2007)

15. Hatakenaka, E., Nosaka, T.: Some topological aspects of 4-fold symmetric quandle invariants of 3-manifolds. Int. J. Math. (to appear)

16. Inoue, A.: Knot quandles and infinite cyclic covering spaces. Kodai. Math. J. 33, 116-122 (2010)

17. Litherland, R.A., Nelson, S.: The Betti numbers of some finite racks. J. Pure Appl. Algebra 178, 187-202 (2003)

18. McCleary, J.: A user's guide to spectral sequences, 2nd edn. Cambridge Studies in Advanced Mathematics, vol. 58. Cambridge University Press, Cambridge (2001)

19. Mochizuki, T.: Some calculations of cohomology groups of finite Alexander quandles. J. Pure Appl. Algebra 179, 287-330 (2003)

20. Mochizuki, T.: The 3-cocycles of the Alexander quandles $\mathbb{F}_{q}[T] /(T-\omega)$. Algebraic Geom. Topol. 5, 183-205 (2005) 
21. Mochizuki, T.: The third cohomology groups of dihedral quandles. J. Knot Theory Ramifications 20 , 1041-1057 (2010)

22. Milgram, J.R.: The bar construction and abelian $H$-spaces. Illinois J. Math. 11, 242-250 (1967)

23. Nosaka, T.: On homotopy groups of quandle spaces and the quandle homotopy invariant of links. Topol. Appl. 158, 996-1011 (2011)

24. Nosaka, T.: On quandle homology groups of Alexander quandles of prime order. Trans. Am. Math. Soc. (to appear)

25. Niebrzydowski, M., Przytycki, J.H.: Homology of dihedral quandles. J. Pure Appl. Algebra 213, 742-755 (2009)

26. Niebrzydowski, M., Przytycki, J.H.: The second quandle homology of the Takasaki quandle of an odd abelian group is an exterior square of the group. J. Knot Theory Ramifications 20(1), 171-177 (2011)

27. Ohtsuki, T. (ed.): Problems on invariants of knots and 3-manifolds. Geom. Topol. Monogr., vol. 4. Invariants of Knots and 3-Manifolds (Kyoto, 2001), pp. 377-572. Geom. Topol. Publ, Coventry (2002)

28. Sanderson, B.J.: Bordism of links in codimension 2. J. Lond. Math. Soc. 35, 367-376 (1987)

29. Soloviev, A.: Non-unitary set-theoretical solutions to the quantum Yang-Baxter equation. Math. Res. Lett. 7, 577-596 (2000)

30. Soulé, C.: Opérations en K-théorie algébrique. Canad. J. Math. 37, 488-550 (1985)

31. Rourke, C., Sanderson, B.: A new classification of links and some calculation using it. arXiv:math.GT/0006062 